\title{
Trap models with slowly decorrelating observables
}

\author{
Peter Sollich $\dagger$ \\ King's College London, Department of Mathematics, London WC2R 2LS, UK
}

\begin{abstract}
We study the correlation and response dynamics of trap models of glassy dynamics, considering observables that only partially decorrelate with every jump. This is inspired by recent work on a microscopic realization of such models, which found strikingly simple linear out-of-equilibrium fluctuation-dissipation relations in the limit of slow decorrelation. For the Barrat and Mézard model with its entropic barriers we obtain exact results at zero temperature $T$ for arbitrary decorrelation factor $\kappa$. These are then extended to nonzero $T$, where the qualitative scaling behaviour and all scaling exponents can still be found analytically. Unexpectedly, the choice of transition rates (Glauber versus Metropolis) affects not just prefactors but also some exponents. In the limit of slow decorrelation even complete scaling functions are accessible in closed form. The results show that slowly decorrelating observables detect persistently slow out-ofequilibrium dynamics, as opposed to intermittent behaviour punctuated by excursions into fast, effectively equilibrated states.
\end{abstract}

\section{Introduction}

Trap models [1, 2, 3, 4, 5, 6, 7, 8, 9, 10, 11, have been recognized in recent years as powerful models of glassy, non-equilibrium dynamics. They describe the motion of a system through its phase space, simplified to a picture of thermally activated hopping in a landscape of traps of energy $E$. The simplest case is that of mean-field trap models, where all traps are taken as mutually accessible and the rate for a transition between two traps depends only on their energies. An alternative motivation for considering trap models is provided by the problem of diffusion in disordered media; here the traps are located in a physical space of low dimension (say, $d=1,2$ or 3 ) and the spatial organization of the traps has to be accounted for [12, 13, 14, 15].

Our motivation in this paper arises from a recent interesting study of a microscopic realization of trap models on the basis of the number partitioning problem [16, 17]. Each partition of $N$ numbers between two piles can be associated with the state of a system of $N$ Ising spins $s_{i}= \pm 1$, and an energy function is then defined to measure how far from optimal the partition is. The dynamics considered by Junier and Bertin in [17] is that at every step $K$ spins are selected and have their values randomized; the new state is then accepted with a probability given by a Metropolis acceptance factor. Junier and Bertin argue that this system evolves in a manner analogous to the trap model considered by

$\dagger$ Email peter.sollich@kcl.ac.uk 
Barrat and Mézard [2]: a new state or trap is selected essentially at random at every step, and accepted according to the Metropolis (or Glauber, see below) probability. To justify the assumption that each new state is effectively random one requires $K \gg 1$; for finite $K$ there is a crossover time, exponentially large in $K$, beyond which this simplification no longer applies 17. In contrast to the usual assumption made in trap models, however, an observable such as the (randomly staggered) magnetization does not decorrelate fully with every transition between states. Instead, it decorrelates by a factor $\kappa=1-K / N$, since only $K$ out of the $N$ spins are updated. Junier and Bertin showed that the consequences of this are rather profound, and most interesting in the limit of slow decorrelation $\kappa \rightarrow 1(K \ll N)$. In particular, they found that fluctuation-dissipation (FD) relations between response and correlation functions had the simple straight-line form expected for systems with well-defined (effective) temperatures [18, 19, 20, 21. The corresponding temperature was equal to the bath temperature $T$ down to half the glass transition temperature $T_{\mathrm{g}}$; for lower $T$, it remained pinned to $T_{\text {eff }}=T_{\mathrm{g}} / 2$. This unusual transition between apparent equilibrium and non-equilibrium dynamics within the glass phase was interpreted as due to a change from activated to entropic slowing down.

Our aim in this paper is to complement the work of [17], which used mainly numerical simulation and simple scaling estimates, with an analytical study of the dynamics of slowly decorrelating observables in trap models. This will allow us, for example, to verify and refine estimates of scaling exponents in [17], but also yield more detailed insights into the nature of the dynamics. In our approach it is also a simple matter to compare different choices of the transition rates (Metropolis and Glauber) and we will see that this has some unexpected consequences, with effects not just on prefactors but also scaling exponents.

To allow a direct comparison with the work of [17] we focus mainly on the Barrat and Mézard model itself; the extension of this to slowly decorrelating observables is defined in Sec. 2. General expressions for correlation and response functions are then derived

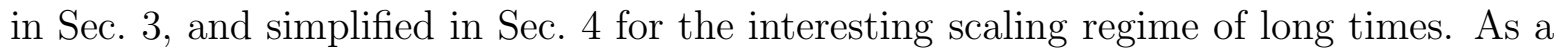
prelude to the analysis of slowly decorrelating observables proper, we consider in Sec. 5 first the standard, fully decorrelating case $\kappa=0$ at nonzero temperature, extending previous analytical results for the FD behaviour in the limit $T \rightarrow 0$ [11. Sec. 6] then looks at arbitrary decorrelation factors $\kappa$, but first at zero temperature, where closed form results can still be obtained. The most general case of $\kappa>0$ and $T>0$ is then analysed in Sec. 7. where we also provide results from numerical solutions of the integral equations for the scaling functions. In Sec. 8, finally, we extend the analysis to more general trap models with a multiplicative dependence of the transition rates on the applied field [10]; this covers in particular Bouchaud's original trap model [1] with its purely activated dynamics. Sec. 9 summarizes the results and discusses the dynamics of slowly decorrelating observables in a wider context. 


\section{Model definition}

Motivated by the arguments discussed in the introduction, we consider a modified Barrat and Mézard model [2] where the magnetizations before and after a jump between two traps are at least partially correlated. Transitions can still take place between arbitrary trap energies, but are more likely between traps with magnetizations that are sufficiently close to each other.

The dynamics of the system is described by the probability $P(E, m, t)$ that the system is in a trap with energy $E$ and magnetization $m$ at time $t$. Of course $m$ is in principle a generic observable, but we continue to use the term magnetization for definiteness. We assume that, starting from a trap with energy $E^{\prime}$ and magnetization $m^{\prime}$, a jump to a new trap with energy $E$ and magnetization $m$ is attempted with probability $\rho(E) \rho\left(m \mid m^{\prime}\right)$. The first factor is simply the density of states of trap energies, reflecting the assumption that transitions between arbitrary energy levels are possible. The factor $\rho\left(m \mid m^{\prime}\right)$, on the other hand, can incorporate various degrees of correlation between $m^{\prime}$ and $m$; if $m$ is typically close to $m^{\prime}$, then decorrelation of $m$ over time is slow. The factorization $\rho(E) \rho\left(m \mid m^{\prime}\right)$ assumes that $E$ and $m$ are selected independently, which in a spin system is a reasonable assumption if $m$ is some staggered magnetization that is uncorrelated with the energy. In a continuous-time description, the time evolution of $P(E, m, t)$ is then governed by the master equation

$$
\begin{aligned}
\frac{\partial}{\partial t} P(E, m, t)= & -\Gamma(E, m) P(E, m, t)+ \\
& +\int d E^{\prime} d m^{\prime} \rho(E) \rho\left(m \mid m^{\prime}\right) w\left(E-E^{\prime}-h m+h m^{\prime}\right) P\left(E^{\prime}, m^{\prime}, t\right)
\end{aligned}
$$

Here $w(\Delta E)$ is the probability with which a proposed transition is accepted. In writing the energy change $\Delta E=E-h m-\left(E^{\prime}-h m^{\prime}\right)$ on which this depends we have allowed for the presence of a field $h$ thermodynamically conjugate to $m$, in order to be able to deduce response properties of $m$. We consider primarily the Glauber form $w(\Delta E)=1 /[1+\exp (\beta \Delta E)]$ of the acceptance probability that was used in previous theoretical studies [2, 11, 22], but also compare with the Metropolis choice, $w(\Delta E)=1$ for $\Delta E<0$ and $w(\Delta E)=\exp (-\beta \Delta E)$ for $\Delta E \geq 0$, which is common in simulations [17, 22]. Here and throughout $\beta=1 / T$ denotes the inverse temperature. In the first term on the right of (1), finally, we have defined

$$
\Gamma(E, m)=\int d E^{\prime} d m^{\prime} \rho\left(E^{\prime}\right) \rho\left(m^{\prime} \mid m\right) w\left(E^{\prime}-E-h m^{\prime}+h m\right)
$$

which can be thought of as the total rate of leaving a trap with energy $E$ and magnetization $m$.

Our dynamics should be capable of describing thermodynamic equilibrium at high temperatures, and thus obey detailed balance. In line with our assumption of independence of energies and magnetizations in an attempted jump, let us assume the overall density of states also factorizes into $\rho(E) \rho(m)$. It is then easy to check that the dynamics (11) obeys detailed balance as long as $\rho\left(m^{\prime} \mid m\right) \rho(m)$ is symmetric under interchange of $m^{\prime}$ and $m$. To be specific, we take $\rho(m)$ to be a Gaussian with zero 
mean and unit variance, and $\rho\left(m^{\prime} \mid m\right)$ a Gaussian with mean $\kappa m$ and variance $1-\kappa^{2}$. (Since we will only need the second order statistics of $m$ and $m^{\prime}$, this assumption in fact constitutes no loss of generality.) The original Barrat and Mézard model then corresponds to Glauber dynamics with $\kappa=0$, while in the opposite limit $\kappa=1$ the magnetization remains frozen to its initial value. For intermediate values, $m$ decorrelates by a factor $\kappa$ with each jump, and we expect the interesting behaviour seen by Junier and Bertin [17] to occur in the limit of slow decorrelation $\kappa \rightarrow 1$ (but keeping $\kappa<1$ ).

\section{General expressions for correlation and response}

For the calculation of the correlation function of $m$, the field can be set to zero. The master equation (11) then simplifies to

$\frac{\partial}{\partial t} P(E, m, t)=-\Gamma(E) P(E, m, t)+\int d E^{\prime} d m^{\prime} \rho(E) \rho\left(m \mid m^{\prime}\right) w\left(E-E^{\prime}\right) P\left(E^{\prime}, m^{\prime}, t\right)$

with

$$
\Gamma(E)=\int d E^{\prime} \rho\left(E^{\prime}\right) w\left(E^{\prime}-E\right)
$$

The propagator $P\left(E, m, t-t_{\mathrm{w}} \mid E_{\mathrm{w}}, m_{\mathrm{w}}\right)$ obeys this equation with the initial condition $P\left(E, m, 0 \mid E_{\mathrm{w}}, m_{\mathrm{w}}\right)=\delta\left(E-E_{\mathrm{w}}\right) \delta\left(m-m_{\mathrm{w}}\right)$ and gives the correlation function as

$C\left(t, t_{\mathrm{w}}\right)=\int d E d m d E_{\mathrm{w}} d m_{\mathrm{w}} m m_{\mathrm{w}} P\left(E, m, t-t_{\mathrm{w}} \mid E_{\mathrm{w}}, m_{\mathrm{w}}\right) \rho\left(m_{\mathrm{w}}\right) P\left(E_{\mathrm{w}}, t_{\mathrm{w}}\right)$

Here we have used that, for $h=0, P\left(E_{\mathrm{w}}, m_{\mathrm{w}}, t_{\mathrm{w}}\right)=\rho\left(m_{\mathrm{w}}\right) P\left(E_{\mathrm{w}}, t_{\mathrm{w}}\right)$; this is true as long as the initial condition has the same structure, e.g. for a quench from thermal equilibrium at high temperature. From (3), the function $\mu\left(E, t-t_{\mathrm{w}} \mid E_{\mathrm{w}}, m_{\mathrm{w}}\right)=$ $\int d m m P\left(E, m, t-t_{\mathrm{w}} \mid E_{\mathrm{w}}, m_{\mathrm{w}}\right)$ obeys, using $\int d m m \rho\left(m \mid m^{\prime}\right)=\kappa m^{\prime}$,

$\frac{\partial}{\partial t} \mu\left(E, t \mid E_{\mathrm{w}}, m_{\mathrm{w}}\right)=-\Gamma(E) \mu\left(E, t \mid E_{\mathrm{w}}, m_{\mathrm{w}}\right)+\kappa \int d E^{\prime} \rho(E) w\left(E-E^{\prime}\right) \mu\left(E^{\prime}, t \mid E_{\mathrm{w}}, m_{\mathrm{w}}\right)$

Because this equation is linear, the factor $m_{\mathrm{w}}$ from the initial condition $\mu\left(E, 0 \mid E_{\mathrm{w}}, m_{\mathrm{w}}\right)=m_{\mathrm{w}} \delta\left(E-E_{\mathrm{w}}\right)$ pulls through and we can write $\mu\left(E, t \mid E_{\mathrm{w}}, m_{\mathrm{w}}\right)=$ $m_{\mathrm{w}} \mu\left(E, t \mid E_{\mathrm{w}}, 1\right) \equiv m_{\mathrm{w}} \mu\left(E, t \mid E_{\mathrm{w}}\right)$, dropping the constant argument 1 . The reduced magnetization $\mu\left(E, t \mid E_{\mathrm{w}}\right)$ then obeys

$\frac{\partial}{\partial t} \mu\left(E, t \mid E_{\mathrm{w}}\right)=-\Gamma(E) \mu\left(E, t \mid E_{\mathrm{w}}\right)+\kappa \int d E^{\prime} \rho(E) w\left(E-E^{\prime}\right) \mu\left(E^{\prime}, t \mid E_{\mathrm{w}}\right)$

with $\mu\left(E, 0 \mid E_{\mathrm{w}}\right)=\delta\left(E-E_{\mathrm{w}}\right)$. For our purposes more useful, however, is the corresponding backward equation,

$\frac{\partial}{\partial t} \mu\left(E, t \mid E_{\mathrm{w}}\right)=-\Gamma\left(E_{\mathrm{w}}\right) \mu\left(E, t \mid E_{\mathrm{w}}\right)+\kappa \int d E^{\prime} \mu\left(E, t \mid E^{\prime}\right) \rho\left(E^{\prime}\right) w\left(E^{\prime}-E_{\mathrm{w}}\right)$

This is because the correlation function can be written as

$$
C\left(t, t_{\mathrm{w}}\right)=\int d E_{\mathrm{w}} M\left(t-t_{\mathrm{w}} \mid E_{\mathrm{w}}\right) P\left(E_{\mathrm{w}}, t_{\mathrm{w}}\right)
$$

with

$$
M\left(t-t_{\mathrm{w}} \mid E_{\mathrm{w}}\right)=\int d E \mu\left(E, t-t_{\mathrm{w}} \mid E_{\mathrm{w}}\right)
$$


By integrating (8) we see that this obeys

$$
\frac{\partial}{\partial t} M\left(t \mid E_{\mathrm{w}}\right)=-\Gamma\left(E_{\mathrm{w}}\right) M\left(t \mid E_{\mathrm{w}}\right)+\kappa \int d E^{\prime} M\left(t \mid E^{\prime}\right) \rho\left(E^{\prime}\right) w\left(E^{\prime}-E_{\mathrm{w}}\right)
$$

with $M\left(0 \mid E_{\mathrm{w}}\right)=1$; the forward equation (7), on the other hand, would yield an expression for $\partial M / \partial t$ which still involves $\mu\left(E, t \mid E_{\mathrm{w}}\right)$. The physical meaning of $M\left(t-t_{\mathrm{w}} \mid E_{\mathrm{w}}\right)$ is as follows: if we start in a state with energy $E_{\mathrm{w}}$ and magnetization $m_{\mathrm{w}}$, then $m_{\mathrm{w}} M\left(t-t_{\mathrm{w}} \mid E_{\mathrm{w}}\right)$ is the average magnetization a time $t-t_{\mathrm{w}}$ later.

For the case $\kappa=0$ the solution of (8) and (11) is trivial,

$$
\begin{aligned}
& \mu\left(E, t \mid E_{\mathrm{w}}\right)=\delta\left(E-E_{\mathrm{w}}\right) \exp \left(-\Gamma\left(E_{\mathrm{w}}\right) t\right) \\
& M\left(t \mid E_{\mathrm{w}}\right)=\exp \left(-\Gamma\left(E_{\mathrm{w}}\right) t\right)
\end{aligned}
$$

and one retrieves the standard result for the hopping correlation function [2, 10, 11, 22, For $\kappa>0$, the Laplace transform (LT) of (8) can be easier to work with; with $s$ conjugate to $t$, this reads

$$
[s+\Gamma(E)] \hat{\mu}\left(E, s \mid E_{\mathrm{w}}\right)-\delta\left(E-E_{\mathrm{w}}\right)=\kappa \int d E^{\prime} \rho(E) w\left(E-E^{\prime}\right) \hat{\mu}\left(E^{\prime}, s \mid E_{\mathrm{w}}\right)
$$

By the same reasoning that lead to (9), the response to a field impulse of amplitude $h$ and duration $\Delta t$, applied at time $t_{\mathrm{w}}$, can be written as

$$
h \Delta t R\left(t, t_{\mathrm{w}}\right)=\int d E d m m M\left(t-t_{\mathrm{w}} \mid E\right) P\left(E, m, t_{\mathrm{w}}+\Delta t\right)
$$

The change in $P\left(E, m, t_{\mathrm{w}}+\Delta t\right)$ from its value without the field (the latter does not contribute to $R$ because it is symmetric in $m$ ) is

$$
\begin{aligned}
\Delta P\left(E, m, t_{\mathrm{w}}+\Delta t\right)= & \Delta t \int d E_{\mathrm{w}} d m_{\mathrm{w}} \times \\
& {\left[\rho(E) \rho\left(m \mid m_{\mathrm{w}}\right) \Delta w\left(E-E_{\mathrm{w}}-h m+h m_{\mathrm{w}}\right) \rho\left(m_{\mathrm{w}}\right) P\left(E_{\mathrm{w}}, t_{\mathrm{w}}\right)\right.} \\
& \left.-\rho\left(E_{\mathrm{w}}\right) \rho\left(m_{\mathrm{w}} \mid m\right) \Delta w\left(E_{\mathrm{w}}-E-h m_{\mathrm{w}}+h m\right) \rho(m) P\left(E, t_{\mathrm{w}}\right)\right]
\end{aligned}
$$

where $\Delta w\left(E-E_{\mathrm{w}}-h m+h m_{\mathrm{w}}\right)=w\left(E-E_{\mathrm{w}}-h m+h m_{\mathrm{w}}\right)-w\left(E-E_{\mathrm{w}}\right)$ is the change of the acceptance probability caused by the field. Expanding this to linear order in $h$ as $\Delta w\left(E-E_{\mathrm{w}}-h m+h m_{\mathrm{w}}\right)=h\left(m_{\mathrm{w}}-m\right) w^{\prime}\left(E-E_{\mathrm{w}}\right)$ and carrying out the integrations over $m$ and $m_{\mathrm{w}}$ gives

$$
\begin{aligned}
R\left(t, t_{\mathrm{w}}\right)= & (1-\kappa) \int d E d E_{\mathrm{w}} M\left(t-t_{\mathrm{w}} \mid E\right) \times \\
& \times\left[-w^{\prime}\left(E-E_{\mathrm{w}}\right) \rho(E) P\left(E_{\mathrm{w}}, t_{\mathrm{w}}\right)-w^{\prime}\left(E_{\mathrm{w}}-E\right) \rho\left(E_{\mathrm{w}}\right) P\left(E, t_{\mathrm{w}}\right)\right]
\end{aligned}
$$

Note that $w^{\prime}(\cdot)$ is negative since the acceptance probability $w(\cdot)$ decreases with increasing energy change; thus both terms in the expression for the response are positive. Explicitly, we have for the Glauber case $-w^{\prime}(\Delta E)=\beta \exp (\beta \Delta E) /[1+\exp (\beta \Delta E)]^{2}$ and for Metropolis $-w^{\prime}(\Delta E)=\Theta(\Delta E) \beta \exp (-\beta \Delta E)$, with $\Theta(\cdot)$ the usual Heaviside step function.

Summarizing, to calculate the correlation and response we need $P\left(E, t_{\mathrm{w}}\right)$, i.e. the solution of the original Barrat and Mézard model without a field, and $M\left(t-t_{\mathrm{w}} \mid E_{\mathrm{w}}\right)$ from (11). The dependence on $\kappa$ is only through the latter. 


\section{Long-time scaling}

From now on we consider mostly an exponential density of trap energies, $\rho(E)=\exp (E)$ for $E<0$; the glass transition temperature is then $T_{\mathrm{g}}=1$. We will also focus on the long-time scaling limit where memory of the initial conditions is lost and typical trap depths are large, $|E| \gg 1$. The exit rate from such deep traps is

$\Gamma(E)=\int_{-\infty}^{0} d E^{\prime} \frac{e^{E^{\prime}}}{1+e^{\beta\left(E^{\prime}-E\right)}}=e^{E} \int_{0}^{e^{-E}} \frac{d z}{1+z^{\beta}} \approx e^{E} \int_{0}^{\infty} \frac{d z}{1+z^{\beta}}=c e^{E}$

with $c=\pi T / \sin (\pi T)$. For Metropolis rates one has similarly

$\Gamma(E)=\int_{-\infty}^{E} d E^{\prime} e^{E^{\prime}}+\int_{E}^{0} d E^{\prime} e^{E^{\prime}} e^{-\beta\left(E^{\prime}-E\right)} \approx \int_{-\infty}^{E} d E^{\prime} e^{E^{\prime}}+\int_{E}^{\infty} d E^{\prime} e^{E^{\prime}} e^{-\beta\left(E^{\prime}-E\right)}$

giving $\Gamma(E)=c^{\mathrm{M}} e^{E}$ with

$$
c^{\mathrm{M}}=1+\frac{1}{\beta-1}=\frac{\beta}{\beta-1}=\frac{1}{1-T}
$$

If the timescales in the system are set by its age at long times, then typical values of $\Gamma(E)$ - and therefore of $e^{E}$ - at time $t$ should be of order $t^{-1}$. This suggests the scaling ansatz $P(E, t)=e^{E} t \mathcal{P}\left(e^{E} t\right)$ where $\mathcal{P}(\omega)$ is the normalized probability distribution of $\omega=e^{E} t$. The zero-field master equation (3) for $P(E, m, t)$ gives after integration over $m$

$$
\frac{\partial}{\partial t} P(E, t)=-\Gamma(E) P(E, t)+\int d E^{\prime} \rho(E) w\left(E-E^{\prime}\right) P\left(E^{\prime}, t\right)
$$

Inserting the scaling form for $P$ and neglecting the upper cutoff $E^{\prime}=0$ in the integral transforms this into an integro-differential version for $\mathcal{P}(\omega)$,

$$
\omega \mathcal{P}^{\prime}(\omega)=-(1+c \omega) \mathcal{P}(\omega)+\int d \omega^{\prime} \frac{\mathcal{P}\left(\omega^{\prime}\right)}{1+\left(\omega / \omega^{\prime}\right)^{\beta}}
$$

For Metropolis rates one only needs to replace $c$ by $c^{\mathrm{M}}$ and $\left[1+\left(\omega / \omega^{\prime}\right)^{\beta}\right]^{-1}$ by $\min \left\{\left(\omega / \omega^{\prime}\right)^{-\beta}, 1\right\}$, and all statements for Glauber rates below can be translated to the Metropolis case in an analogous way unless specified otherwise.

From (23) it follows directly for $\omega \rightarrow 0$ that $\mathcal{P}(0)=1$, as also found by Bertin [22] who used $1 / \omega$ as the scaling variable. For large $\omega$, on the other hand, i.e. relatively shallow traps, one expects effective equilibration and therefore $\mathcal{P}(\omega) \sim e^{-\beta E} \sim \omega^{-\beta}$. (There is no density of states factor here because the density of states with respect to $e^{E}$ is uniform on the allowed range $0<e^{E}<1$.) At $T=0$ this power-law tail becomes an exponential as we will see below. For a numerical solution at $T>0$, it is useful to rewrite (23) as

$$
\omega \mathcal{P}(\omega)=\int_{0}^{\omega} d \omega^{\prime} f\left(\omega^{\prime}\right) e^{-c\left(\omega-\omega^{\prime}\right)}, \quad f(\omega)=\int d \omega^{\prime} \frac{\mathcal{P}\left(\omega^{\prime}\right)}{1+\left(\omega / \omega^{\prime}\right)^{\beta}}
$$

For later we also note the following relation between successive moments of $\mathcal{P}(\omega)$, obtained by multiplying (23) with $\omega^{n}(-1<n<\beta-1)$ and integrating over $\omega$ :

$$
\frac{\int d \omega \omega^{n+1} \mathcal{P}(\omega)}{\int d \omega \omega^{n} \mathcal{P}(\omega)}=n\left(c-\int d z \frac{z^{n}}{1+z^{\beta}}\right)^{-1}=\frac{n}{c-c_{n}}
$$


The constant $c_{n}$ in this expression generalizes $c \equiv c_{0}$ :

$$
c_{n}=\int d z \frac{z^{n}}{1+z^{\beta}}=\frac{\pi T}{\sin [\pi T(n+1)]}
$$

For Metropolis rates the analogous expression for the moment ratio (25) is $n /\left(c^{\mathrm{M}}-c_{n}^{\mathrm{M}}\right)$ with

$$
c_{n}^{\mathrm{M}}=\int d z z^{n} \min \left\{z^{-\beta}, 1\right\}=\frac{\beta}{(n+1)(\beta-n-1)}
$$

We next turn to the long-time behaviour of $\mu\left(E, t-t_{\mathrm{w}} \mid E_{\mathrm{w}}\right)$. By arguments similar to those above, this should have the scaling form

$$
\mu\left(E, t-t_{\mathrm{w}} \mid E_{\mathrm{w}}\right)=\gamma \mathcal{U}(\gamma, \tau), \quad \gamma=e^{E} / e^{E_{\mathrm{w}}}, \quad \tau=\left(t-t_{\mathrm{w}}\right) e^{E_{\mathrm{w}}}
$$

where $\mathcal{U}(\gamma, \tau)$ is the normalized distribution of $\gamma$ after the rescaled time-interval $\tau$. The scaling form for $M$ then follows directly as

$M\left(t-t_{\mathrm{w}} \mid E_{\mathrm{w}}\right)=\int d E \mu\left(E, t-t_{\mathrm{w}} \mid E_{\mathrm{w}}\right)=\mathcal{M}(\tau), \quad \mathcal{M}(\tau)=\int d \gamma \mathcal{U}(\gamma, \tau)$

The dependence on $\tau=\left(t-t_{\mathrm{w}}\right) e^{E_{\mathrm{w}}}$ makes sense because, for $\left|E_{\mathrm{w}}\right| \gg 1, e^{E_{\mathrm{w}}}$ sets the scale of the exit rate from traps of depth $E_{\mathrm{w}}$. The dynamical equation (11) becomes in the scaling regime

$$
\mathcal{M}^{\prime}(\tau)=-c \mathcal{M}(\tau)+\kappa \int \frac{d \tau^{\prime}}{\tau} \frac{\mathcal{M}\left(\tau^{\prime}\right)}{1+\left(\tau^{\prime} / \tau\right)^{\beta}}
$$

This is similar in form to (23) for $\mathcal{P}(\omega)$; accordingly, there is again an alternative version suitable for numerical iteration,

$$
\mathcal{M}(\tau)=e^{-c \tau}+\kappa \int_{0}^{\tau} d \tau^{\prime} g\left(\tau^{\prime}\right) e^{-c\left(\tau-\tau^{\prime}\right)}, \quad g(\tau)=\int \frac{d \tau^{\prime}}{\tau} \frac{\mathcal{M}\left(\tau^{\prime}\right)}{1+\left(\tau^{\prime} / \tau\right)^{\beta}}
$$

The scaling of the correlation and response functions can now be deduced. For the correlation (91) one gets

$$
C\left(t, t_{\mathrm{w}}\right)=\mathcal{C}\left(\left(t-t_{\mathrm{w}}\right) / t_{\mathrm{w}}\right), \quad \mathcal{C}(x)=\int d \omega \mathcal{M}(x \omega) \mathcal{P}(\omega)
$$

which displays the expected simple aging scaling with $x=\left(t-t_{\mathrm{w}}\right) / t_{\mathrm{w}}$, implying that relaxation time scales grow linearly with the age. Similarly, the scaling form of the response function (18) is $R\left(t, t_{\mathrm{w}}\right)=t_{\mathrm{w}}^{-1} \mathcal{R}(x)$ with

$$
\begin{aligned}
\operatorname{TR}(x) & =(1-\kappa) \int d \omega \mathcal{M}(x \omega) r(\omega) \\
r(\omega) & =\int d \omega^{\prime} \frac{\mathcal{P}(\omega)+\mathcal{P}\left(\omega^{\prime}\right)}{\left[1+\left(\omega / \omega^{\prime}\right)^{\beta}\right]\left[1+\left(\omega^{\prime} / \omega\right)^{\beta}\right]} \\
& =\operatorname{Tc} \omega \mathcal{P}(\omega)+\int d \omega^{\prime} \frac{\mathcal{P}\left(\omega^{\prime}\right)}{\left[1+\left(\omega / \omega^{\prime}\right)^{\beta}\right]\left[1+\left(\omega^{\prime} / \omega\right)^{\beta}\right]}
\end{aligned}
$$

where we have used the integral

$$
\int \frac{d \omega^{\prime}}{\left[1+\left(\omega / \omega^{\prime}\right)^{\beta}\right]\left[1+\left(\omega^{\prime} / \omega\right)^{\beta}\right]}=\omega \int \frac{d z z^{\beta}}{\left(1+z^{\beta}\right)^{2}}=T \omega \int d z z\left(-\frac{d}{d z}\right) \frac{1}{1+z^{\beta}}=T c \omega
$$


For Metropolis rates, one has similarly

$$
\begin{aligned}
r(\omega) & =\int d \omega^{\prime}\left[\mathcal{P}(\omega) \Theta\left(\omega^{\prime}-\omega\right)\left(\omega^{\prime} / \omega\right)^{-\beta}+\mathcal{P}\left(\omega^{\prime}\right) \Theta\left(\omega-\omega^{\prime}\right)\left(\omega / \omega^{\prime}\right)^{-\beta}\right] \\
& =T c^{\mathrm{M}} \omega \mathcal{P}(\omega)+\int^{\omega} d \omega^{\prime} \mathcal{P}\left(\omega^{\prime}\right)\left(\omega / \omega^{\prime}\right)^{-\beta}
\end{aligned}
$$

We will need the asymptotic behaviour of $r(\omega)$ below. For small $\omega$, one can approximate $\mathcal{P}(\omega)$ in (34) by 1 . But the denominator ensures that $\omega$ and $\omega^{\prime}$ are of the same order, so the same argument can be applied to $\mathcal{P}\left(\omega^{\prime}\right)$. This gives

$$
r(\omega)=2 T c \omega
$$

in the limit of small $\omega$; for Metropolis rates one finds $r(\omega)=2 T c^{\mathrm{M}} \omega$ instead. For large $\omega$, on the other hand, where $\mathcal{P}(\omega) \sim \omega^{-\beta}$, one has

$r(\omega)=\mathcal{P}(\omega) \int d \omega^{\prime} \frac{1+\left(\omega / \omega^{\prime}\right)^{\beta}}{\left[1+\left(\omega / \omega^{\prime}\right)^{\beta}\right]\left[1+\left(\omega^{\prime} / \omega\right)^{\beta}\right]}=\mathcal{P}(\omega) \int d \omega^{\prime} \frac{1}{1+\left(\omega^{\prime} / \omega\right)^{\beta}}=c \omega \mathcal{P}(\omega)$

giving the scaling $r(\omega) \sim \omega^{1-\beta}$; the Metropolis case again differs only by $c \rightarrow c^{\mathrm{M}}$.

The susceptibility or step response $\chi\left(t, t_{\mathrm{w}}\right)=\int_{t_{\mathrm{w}}}^{t} d t^{\prime} R\left(t, t^{\prime}\right)$ follows from (33) as

$$
\chi\left(t, t_{\mathrm{w}}\right)=\int_{t_{\mathrm{w}}}^{t} d t^{\prime} \frac{1}{t^{\prime}} \mathcal{R}\left(\left(t-t^{\prime}\right) / t^{\prime}\right)=\chi(x), \quad \chi(x)=\int_{0}^{x} \frac{d x^{\prime}}{1+x^{\prime}} \mathcal{R}\left(x^{\prime}\right)
$$

and again depends only on $x=\left(t-t_{\mathrm{w}}\right) / t_{\mathrm{w}}$, exhibiting simple aging scaling. Finally, the fluctuation-dissipation ratio (FDR) scales in the same manner,

$$
X\left(t, t_{\mathrm{w}}\right)=\frac{T R\left(t, t_{\mathrm{w}}\right)}{\left(\partial / \partial t_{\mathrm{w}}\right) C\left(t, t_{\mathrm{w}}\right)}=\mathcal{X}(x), \quad \mathcal{X}(x)=-\frac{T \mathcal{R}(x)}{(1+x) \mathcal{C}^{\prime}(x)}
$$

We recall that $X=1$ corresponds to equilibrium, where the fluctuation-dissipation theorem holds; out of equilibrium, it then makes sense to define $T_{\text {eff }}=T / X$ as an

effective temperature [18, 19, 20, 21]. This quantity allows a straightforward physical interpretation only when it is time-independent, at least within a given time-sector; the one of interest here is $t-t_{\mathrm{w}} \sim t_{\mathrm{w}}$. Since the definition (42) can be written as $X=-\left(\partial T \chi / \partial t_{\mathrm{w}}\right) /\left(\partial C / \partial t_{\mathrm{w}}\right)$, such time-independence corresponds to a straight-line FD plot of $\chi\left(t, t_{\mathrm{w}}\right)$ versus $C\left(t, t_{\mathrm{w}}\right)$. More generally, if $t_{\mathrm{w}}$ is used as the parameter varying along the curve [9, 23, 24] then $X / T$ is the negative slope of such an FD plot.

\section{Standard Barrat and Mézard model at $T>0$}

We first consider the case $\kappa=0$, i.e. the standard Barrat and Mézard model, at arbitrary temperature $T\left(<T_{\mathrm{g}}=1\right)$ and with an exponential density of states. One then has, from (13), (19) and (29), $\mathcal{M}(\tau)=\exp (-c \tau)$. The scaling forms (32) and (33) of correlation and response function thus simplify to

$$
\mathcal{C}(x)=\int d \omega e^{-c x \omega} \mathcal{P}(\omega)
$$

and

$$
T \mathcal{R}(x)=\int d \omega e^{-c x \omega} r(\omega)
$$


From this the large- $x$ asymptotics can be obtained directly: only the region $\omega \sim 1 / x$ contributes to the integrals so that in $\mathcal{C}(x)$ we can set $\mathcal{P}(\omega) \approx \mathcal{P}(0)=1$, giving

$$
\mathcal{C}(x)=\frac{1}{c x}
$$

as found previously by Bertin [22]. For $\mathcal{R}(x)$, using (39) leads similarly to

$$
T \mathcal{R}(x)=\frac{2 T}{c x^{2}}
$$

The FDR from (42) thus behaves asymptotically as $\mathcal{X}(x)=2 T / x$. In terms of $C$ this gives $X=-T d \chi / d C=2 T c C$ for small $C$. The limiting FD plot therefore always starts parabolically at the top, $\chi(C)=\chi(0)-c C^{2}$ for $C \rightarrow 0$. The FDR itself decays to zero asymptotically, $\mathcal{X}(x \rightarrow \infty)=0$ for all $T<T_{\mathrm{g}}$, as in the case of the Bouchaud trap model [4, 9].

More interesting is the short-time FDR $\mathcal{X}(x \rightarrow 0)$. Naively, this is from (42)

$$
\mathcal{X}(x \rightarrow 0)=\frac{T \mathcal{R}(0)}{-\mathcal{C}^{\prime}(0)}=\frac{\int d \omega r(\omega)}{\int d \omega c \omega \mathcal{P}(\omega)}=\frac{2 T c \int d \omega \omega \mathcal{P}(\omega)}{c \int d \omega \omega \mathcal{P}(\omega)}=2 T
$$

using (43) and (44) with $x=0$ as well as (35) and (36) (or (38) for the Metropolis case). The effective temperature associated with this FDR is therefore $T_{\text {eff }}=1 / 2$, independently of $T$. However, this conclusion only holds for $T<1 / 2$. For larger $T$, the integral

$$
-\mathcal{C}^{\prime}(x)=\int d \omega c \omega e^{-c x \omega} \mathcal{P}(\omega)
$$

is actually divergent for $x=0$ because of the $\omega^{-\beta}$ tail of $\mathcal{P}(\omega)$. For small but nonzero $x$, the exponential acts as a cutoff at $\omega \sim 1 / x$ so that $-\mathcal{C}^{\prime}(x) \sim(1 / x)^{2-\beta}=x^{\beta-2}$, in agreement with the short-time singularity in $C$ identified in $[22$. The response $T \mathcal{R}(x)=\int d \omega e^{-c x \omega} r(\omega)$ has the same small- $x$ singularity because $r(\omega) \sim \omega^{1-\beta}$ for $\omega \rightarrow \infty$. In fact, for small $x$ one can replace $r(\omega)$ by its asymptotic form (40) to get

$$
T \mathcal{R}(x)=\int d \omega c \omega e^{-c x \omega} P(\omega)=-\mathcal{C}^{\prime}(x)
$$

This shows that the short-time FDR is, for $T>1 / 2$,

$$
\mathcal{X}(x \rightarrow 0)=1
$$

In this temperature-range the FD relation between $\chi$ and $C$ is therefore always of a pseudo-equilibrium form in its initial part, with a (negative) slope equal to $1 / T$. This matches continuously with the constant slope of $1 / T_{\text {eff }}=2$ found above for $T<1 / 2$.

Intuitively, one can understand the occurrence of pseudo-equilibrium behaviour for $T>1 / 2$ by looking at the average hopping rate. This is

$$
\Gamma(t)=\int d E \Gamma(E) P(E, t)=t^{-1} \int d \omega c \omega \mathcal{P}(\omega)
$$

where the second form applies in the scaling regime. For $T<1 / 2$, the integral converges and $\Gamma(t) \sim 1 / t$ as one would naively expect from our scaling assumption: typical relaxation times are $\sim t$, thus typical rates are $\sim 1 / t$. For $T>1 / 2$, however, the integral is divergent at the upper end and one has to take into account the cutoff at 


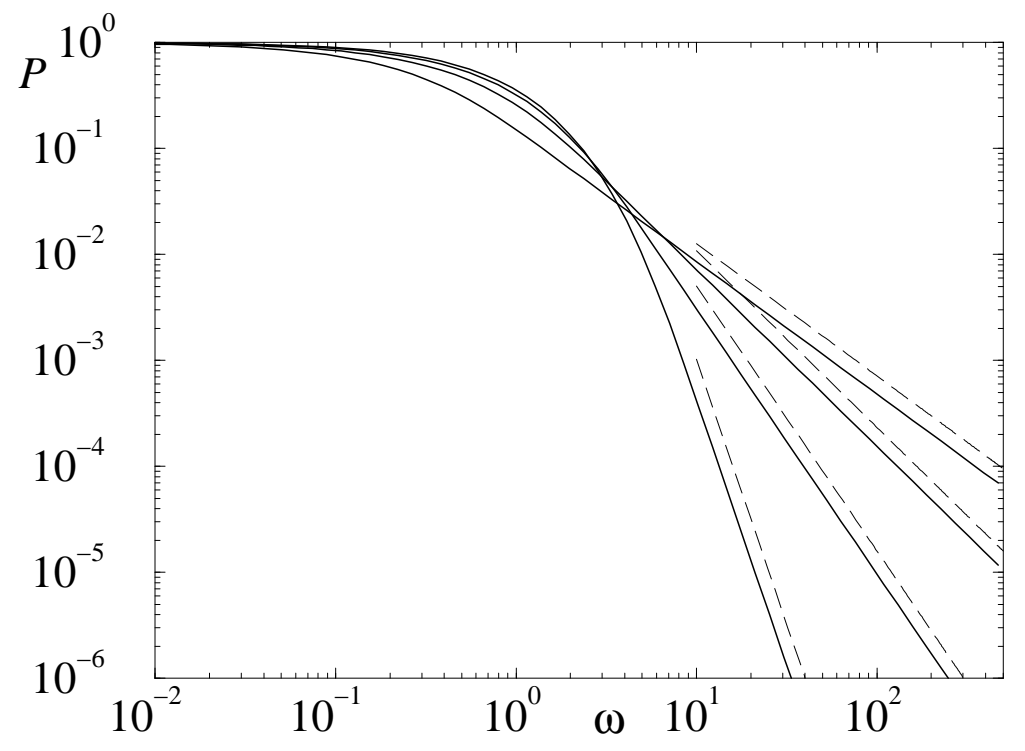

Figure 1. Numerically calculated scaling distributions $\mathcal{P}(\omega)$ for Glauber dynamics at $T=0.2,0.4,0.6,0.8$ (bottom to top on the right). The dashed lines indicate the expected asymptotic scaling $\mathcal{P}(\omega) \sim \omega^{-\beta}$.

$E=0$, corresponding to $\omega=t$, leading to $\Gamma(t) \sim t^{1-\beta}$. This is entirely dominated by the very small probability of being in shallow traps with atypically fast relaxation rates of $\mathcal{O}(1)$. Now the response $R\left(t, t_{\mathrm{w}}\right)$ and the initial decay $\left(\partial C / \partial t_{\mathrm{w}}\right)\left(t, t_{\mathrm{w}}\right)$ of the correlation function are sensitive only to hops taking place between $t_{\mathrm{w}}$ and $t$. For small $t-t_{\mathrm{w}}$ these are precisely the same events that dominate $\Gamma(t)$. Since they are in the "effective equilibrium" tail of $\mathcal{P}(\omega)$, it is then not surprising to find a pseudo-equilibrium form of the FD relation.

Fig. 11 shows some numerically calculated scaling distributions $\mathcal{P}(\omega)$ for Glauber rates; these exhibit the expected asymptotic behaviour $\sim \omega^{-\beta}$ for large $\omega$. To avoid end effects in the iterative numerical solution of (24), we stored not $\mathcal{P}$ and $f$ themselves but $\mathcal{P}(\omega)(1+\omega)^{\beta}$ and similarly $f(\omega)(1+\omega)^{\beta-1}$. These functions have nonzero limits for $\omega \rightarrow \infty$ (and $\omega \rightarrow 0$ ) and so are suitable for evaluating the required integrals over $\omega=0 \ldots \infty$.

Fig. 2 displays the resulting FD plots of $\chi$ vs $C$; these are valid in the limit of long times which we have already taken by working in the scaling regime. (To get reliable results for $\chi(x)$, one has to do the $x^{\prime}$-integration from (41) before the $\omega$-integration in (133); otherwise the singularity of $\mathcal{R}(x)$ for $x \rightarrow 0$ at $T>1 / 2$ leads to problems.) The initial slopes agree well with the theoretical predictions, as shown by the dashed lines. The asymptotic slopes for $C \rightarrow 0$ are likewise consistent with the predicted value of 0 . The inset of the figure explores this region in more detail, showing $(1-\chi) /\left(c C^{2}\right)$. From the analysis above this quantity should converge to 1 for $C \rightarrow 0$. The numerical data are consistent with this, though for $T=0.8$ the approach to the limit is very slow. This makes sense: as $T$ tends to $T_{\mathrm{g}}=1$ from below, the FD plot approaches a straight line, 


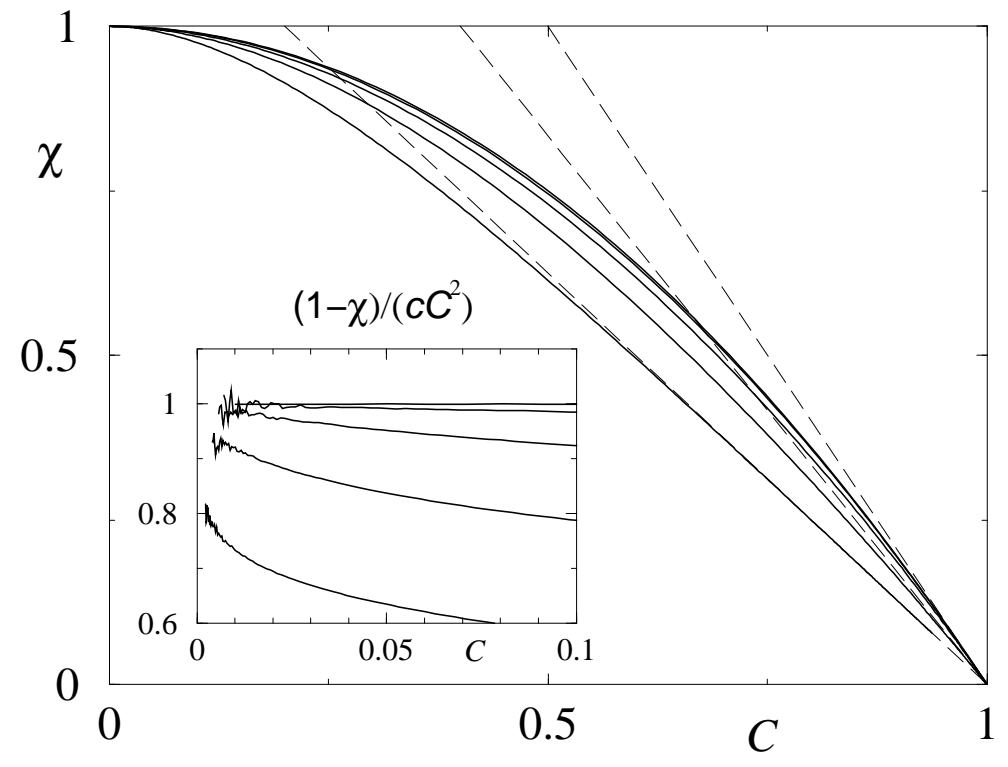

Figure 2. Numerically calculated long-time FD relations $\chi(C)$ in the original Barrat and Mézard model ( $\kappa=0$, Glauber dynamics). Solid lines are for $T=0$ (exact [11]), $0.2,0.4,0.6$ and 0.8 from top to bottom; the results for $T=0$ and $T=0.2$ are almost indistinguishable. The dashed lines show the theoretically predicted initial slopes $(2$ for $T<1 / 2$, and $1 / T$ for $T>1 / 2)$. The inset graphs $(1-\chi) /\left(c C^{2}\right)$ versus $C$; our theory predicts that this quantity converges to 1 for $C \rightarrow 0$.

and so the quadratic expansion around $C=0$ will be valid in a region that shrinks to zero in the limit.

One notable feature of the numerical results is that the asymptotic value $\chi(C=0)$ is equal to 1 for all $T<1$, at least to within our numerical accuracy of around $10^{-5}$. This is as in the Bouchaud model [10], and has been conjectured also for the Barrat and Mézard model, on the same physical grounds [11]: the susceptibility freezes to its value at $T=T_{\mathrm{g}}=1$ as $T$ is decreased below the glass transition. It ought to be possible to confirm this result analytically, but we have not yet found a way of doing this.

It should be noted that the FD plot is not quite $T$-independent for $T<1 / 2$. While such $T$-independence had been suggested by the simulations of [17], the scaling-regime numerics shown in Fig. 2 clearly rule it out. Also, after a little reflection one sees that higher derivatives of $C(x)$ diverge for $x \rightarrow 0$ already at lower temperatures; e.g. $C^{\prime \prime}(x)$ diverges for $T>1 / 3, C^{\prime \prime \prime}(x)$ for $T>1 / 4$ etc.

\section{Slowly decorrelating observables, $T=0$}

In this section and the next we consider slowly decorrelating observables, i.e. $\kappa>0$. We begin by analysing the zero temperature dynamics, where a number of results can be derived for a general density of states $\rho(E)$ and finite times (i.e. without taking the scaling limit). 
Compared to the standard case $\kappa=0$, the additional task is to calculate $\mu\left(E, t-t_{\mathrm{w}} \mid E_{\mathrm{w}}\right)$. At $T=0$, one has $w\left(E-E^{\prime}\right)=\Theta\left(E^{\prime}-E\right)$ and $\Gamma(E)=\int_{-\infty}^{E} d E^{\prime} \rho\left(E^{\prime}\right)$ for both Glauber and Metropolis rates; equation (14) thus simplifies to

$$
\frac{s+\Gamma(E)}{\rho(E)} \hat{\mu}\left(E, s \mid E_{\mathrm{w}}\right)-\frac{1}{\rho\left(E_{\mathrm{w}}\right)} \delta\left(E-E_{\mathrm{w}}\right)=\kappa \int_{E}^{0} d E^{\prime} \hat{\mu}\left(E^{\prime}, s \mid E_{\mathrm{w}}\right)
$$

Differentiating w.r.t. $E$ gives a differential equation for $\mu\left(E, s \mid E_{\mathrm{w}}\right)$ as a function of $E$. This is easily solved, with the result

$$
\begin{aligned}
\hat{\mu}\left(E, s \mid E_{\mathrm{w}}\right) & =-\frac{1}{s+\Gamma\left(E_{\mathrm{w}}\right)} \frac{\partial}{\partial E}\left[\Theta\left(E_{\mathrm{w}}-E\right) \exp \left(\kappa \int_{E}^{E_{\mathrm{w}}} d E^{\prime} \frac{\rho\left(E^{\prime}\right)}{s+\Gamma\left(E^{\prime}\right)}\right)\right] \\
& =-\frac{1}{s+\Gamma\left(E_{\mathrm{w}}\right)} \frac{\partial}{\partial E}\left[\Theta\left(E_{\mathrm{w}}-E\right)\left(\frac{s+\Gamma\left(E_{\mathrm{w}}\right)}{s+\Gamma(E)}\right)^{\kappa}\right]
\end{aligned}
$$

Before we exploit this to obtain $M$ and thence the correlation and response functions, it is worth noting from (77) that, for the limiting case $\kappa=1, \mu\left(E, t-t_{\mathrm{w}} \mid E_{\mathrm{w}}\right)$ is simply the propagator $P\left(E, t-t_{\mathrm{w}} \mid E_{\mathrm{w}}\right)$ of the original Barrat and Mézard model without a field. Inverting the LT in (53) thus yields the exact zero-temperature propagator

$$
P\left(E, t-t_{\mathrm{w}} \mid E_{\mathrm{w}}\right)=-\frac{\partial}{\partial E}\left[\Theta\left(E_{\mathrm{w}}-E\right) e^{-\Gamma(E)\left(t-t_{\mathrm{w}}\right)}\right]
$$

Applying this to $t_{\mathrm{w}}=0$ gives as the general solution starting from an initial distribution $P(E, 0)$

$$
P(E, t)=-\frac{\partial}{\partial E}\left[e^{-\Gamma(E) t} \int_{E}^{0} d E^{\prime} P\left(E^{\prime}, 0\right)\right]
$$

At long times most of the mass of this is a low $E$, where the integral inside the square brackets can be set to one. One thus recovers $P(E, t)=\rho(E) t \exp [-\Gamma(E) t]$ as the long-time scaling form of the distribution over trap energies, independently of the initial distribution [2]. For an exponential density of states, $\rho(E)=e^{E}$, this becomes $P(E, t)=e^{E} t \mathcal{P}\left(e^{E} t\right)$ with the scaling function $\mathcal{P}(\omega)=\exp (-\omega)$. This has an exponential tail as anticipated above, and one easily checks that it solves the $T \rightarrow 0$ limit of (23).

Returning now to the result (53) for general $\kappa$, we obtain for the LT of $M\left(t-t_{\mathrm{w}} \mid E_{\mathrm{w}}\right)$ $\hat{M}\left(s \mid E_{\mathrm{w}}\right)=\int d E \hat{\mu}\left(E, s \mid E_{\mathrm{w}}\right)=\frac{1}{s+\Gamma\left(E_{\mathrm{w}}\right)}\left(\frac{s+\Gamma\left(E_{\mathrm{w}}\right)}{s}\right)^{\kappa}=s^{-\kappa}\left[s+\Gamma\left(E_{\mathrm{w}}\right)\right]^{\kappa-1}$

Inverting the LT gives a hypergeometric function,

$$
M\left(t-t_{\mathrm{w}} \mid E_{\mathrm{w}}\right)={ }_{1} F_{1}\left(1-\kappa, 1 ;-\Gamma\left(E_{\mathrm{w}}\right)\left(t-t_{\mathrm{w}}\right)\right)
$$

If we now specialize to the exponential distribution of trap energies, we have $\Gamma(E)=e^{E}$ and so (57) is exactly of the scaling form (29) discussed above, with

$$
\mathcal{M}(\tau)={ }_{1} F_{1}(1-\kappa, 1 ;-\tau)=\sum_{k=0}^{\infty} \frac{(k-\kappa) !}{(-\kappa) ! k !^{2}}(-\tau)^{k}
$$

(Non-integer factorials are defined in terms of the Gamma function, $a !=\Gamma(a+1)$; we avoid the use of $\Gamma$ for such constants to prevent confusion with our exit rates $\Gamma(E)$.) 
Trap models with slowly decorrelating observables

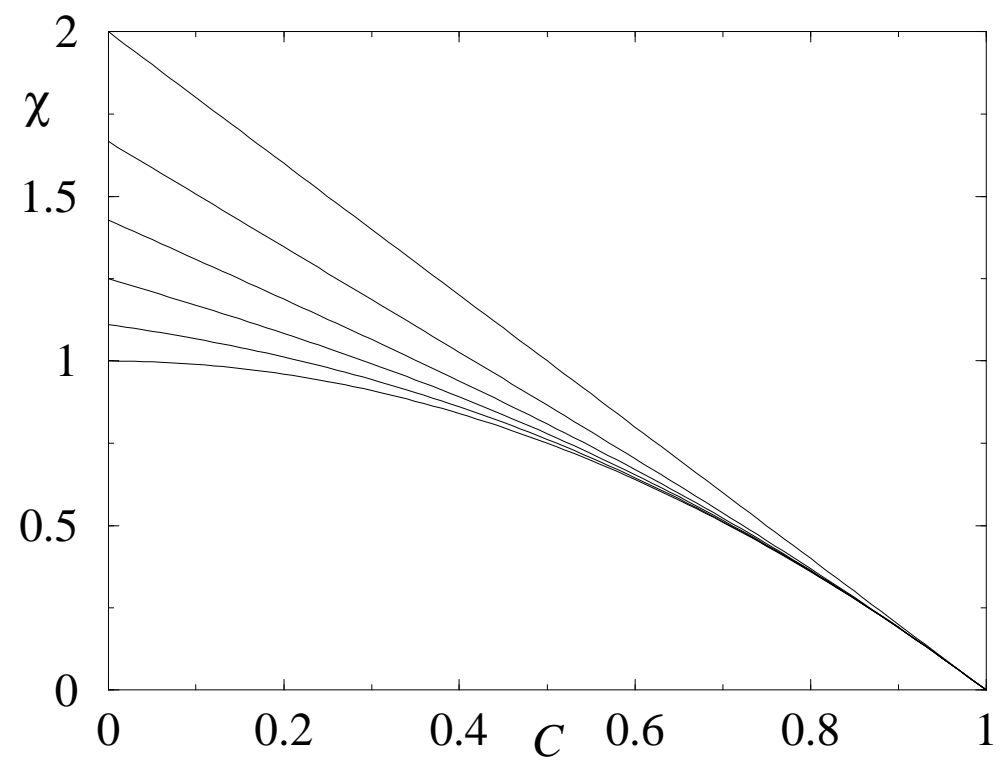

Figure 3. Long-time FD plots $\chi(C)$ at $T=0$, for $\kappa=0,0.2,0.4,0.6,0.8,1$ from bottom to top.

Both of these results can also be obtained directly from the relevant equations (11) and (30) for $M\left(t \mid E_{\mathrm{w}}\right)$ and $\mathcal{M}(\tau)$, by representing these quantities as power series in $t$ and $\tau$, respectively, and determining the coefficients recursively.

From $\mathcal{M}(\tau)$ we get the scaling limit of the correlation function, using $\mathcal{P}(\omega)=$ $\exp (-\omega)$

$$
\mathcal{C}(x)=\int d \omega \mathcal{M}(x \omega) \mathcal{P}(\omega)=\sum_{k=0}^{\infty} \frac{(k-\kappa) !}{(-\kappa) ! k !}(-x)^{k}=(1+x)^{\kappa-1}
$$

so that $C\left(t, t_{\mathrm{w}}\right)$ has the simple long-time form $C\left(t, t_{\mathrm{w}}\right)=\left(t_{\mathrm{w}} / t\right)^{1-\kappa}$. This result can also be derived without recourse to series expansions: denoting $\hat{\mathcal{M}}(\cdot)$ the LT of $\mathcal{M}(\tau)$, we have from (32)

$$
\int d x e^{-\sigma x} \mathcal{C}(x)=\int d \omega \omega^{-1} \hat{\mathcal{M}}(\sigma / \omega) e^{-\omega}=\int d x x^{-1} \hat{\mathcal{M}}\left(x^{-1}\right) e^{-\sigma x}
$$

so that $\mathcal{C}(x)=x^{-1} \hat{\mathcal{M}}\left(x^{-1}\right)$. With $\hat{\mathcal{M}}\left(x^{-1}\right)=\left(x^{-1}\right)^{-\kappa}\left(x^{-1}+1\right)^{\kappa-1}$ from (56) one gets $\mathcal{C}(x)=(1+x)^{\kappa-1}$ as before.

For the response, we note that the denominator factor in (34) tends to $T \omega \delta\left(\omega-\omega^{\prime}\right)$ for $T \rightarrow 0$; the same conclusion also holds in the Metropolis case (37). This gives for the scaling function

$$
\begin{aligned}
\mathcal{R}(x) & =2(1-\kappa) \int d \omega \mathcal{M}(x \omega) \omega \mathcal{P}(\omega)=2(1-\kappa) \sum_{k=0}^{\infty} \frac{(k+1)(k-\kappa) !}{(-\kappa) ! k !}(-x)^{k} \\
& =2(1-\kappa) \frac{\partial}{\partial x}[x \mathcal{C}(x)]=2 \kappa(1-\kappa)(1+x)^{\kappa-1}+2(1-\kappa)^{2}(1+x)^{\kappa-2}
\end{aligned}
$$


For the susceptibility this implies, from (41),

$$
\begin{aligned}
\chi(x) & =2 \kappa\left[1-(1+x)^{\kappa-1}\right]+\frac{2(1-\kappa)^{2}}{2-\kappa}\left[1-(1+x)^{\kappa-2}\right] \\
& =2 \kappa[1-C]+\frac{2(1-\kappa)^{2}}{2-\kappa}\left[1-C^{(2-\kappa) /(1-\kappa)}\right]
\end{aligned}
$$

where in the second equality $x$ has been expressed in terms of $C$ to give the longtime FD relation $\chi(C)$. This is displayed in Fig. 3 for some representative values of $\kappa$. The slope of the FD plot is $-\chi^{\prime}(C)=2 \kappa+2(1-\kappa) C^{1 /(1-\kappa)}$, with initial value $-\chi^{\prime}(C=1)=2$ independently of $\kappa$, corresponding to an "effective short-time temperature" of $T_{\text {eff }}=1 / 2\left(=T_{\mathrm{g}} / 2\right.$ in dimensional units). The asymptotic slope, on the other hand, is $-\chi^{\prime}(C=0)=2 \kappa$ and depends continuously on $\kappa$. In the limit $\kappa \rightarrow 1$ (where $C(x)=(1+x)^{\kappa-1}$ decays very slowly with $x$, and $\chi(x)$ grows correspondingly slowly), the FD plot becomes a straight line of negative slope 2, suggesting that this slowly decorrelating observable measures a well-defined $T_{\text {eff }}=1 / 2$. This result lends support to the straight-line FD plots found in the simulations of Junier and Bertin [17.

For later, we note that by expressing the ratio of factorials in (58) as a Beta function integral and then performing the sum over $k$ one gets an alternative form for $\mathcal{M}(\tau)$,

$$
\mathcal{M}(\tau)=\frac{1}{(-\kappa) !(\kappa-1) !} \int_{0}^{1} d z e^{-z \tau} z^{-\kappa}(1-z)^{\kappa-1}
$$

This implies in particular that $\mathcal{M}(\tau)=\tau^{-(1-\kappa)} /(\kappa-1)$ ! for large $\tau$ and $\kappa>0$.

\section{Slowly decorrelating observables, $T>0$}

Finally we consider the most general case of dynamics at nonzero temperature and observables with incomplete decorrelation $(\kappa>0)$. We focus directly on the scaling limit for a system with an exponential density of states; the key issue is again solving for $\mathcal{M}(\tau)$. For short times this is easy: from (30), $\mathcal{M}^{\prime}(0)=-c+\kappa c$ and thus $\mathcal{M}(\tau)=1-(1-\kappa) c \tau+\mathcal{O}\left(\tau^{2}\right)$. From this the initial FDR for $T<1 / 2$ can be worked out as in (47)

$$
\mathcal{X}(x \rightarrow 0)=\frac{T \mathcal{R}(0)}{-\mathcal{C}^{\prime}(0)}=\frac{(1-\kappa) \int d \omega r(\omega)}{\int d \omega(1-\kappa) c \omega \mathcal{P}(\omega)}=2 T
$$

The $\kappa$-dependent factors $(1-\kappa)$ cancel, so the initial slope of the FD plot remains 2 for all $\kappa$. Metropolis rather than Glauber rates again only replace $c$ by $c^{\mathrm{M}}$ everywhere.

For $T>1 / 2$, the linearization in $\tau$ breaks down as before, and the integrals for $\mathcal{R}(x)$ and $1-\mathcal{C}(x)$ will be dominated by large $\omega \sim 1 / x$ for $x \rightarrow 0$. One can therefore use the asymptotic behaviour of $\mathcal{P}(\omega) \propto \omega^{-\beta}$ and $r(\omega) \propto c \omega^{1-\beta}$ (with the same proportionality constant, according to (40) ) to write

$$
\begin{aligned}
& 1-\mathcal{C}(x) \propto \int d \omega[1-\mathcal{M}(x \omega)] \omega^{-\beta}=x^{\beta-1} \int d \tau[1-\mathcal{M}(\tau)] \tau^{-\beta} \\
& T \mathcal{R}(x) \propto(1-\kappa) c \int d \omega \mathcal{M}(x \omega) \omega^{1-\beta}=(1-\kappa) c x^{\beta-2} \int d \tau \mathcal{M}(\tau) \tau^{1-\beta}
\end{aligned}
$$


These singularities (but not their prefactors) are the same as for $\kappa=0$, and correspondingly the FDR again approaches a nonzero limit for $x \rightarrow 0$ :

$\mathcal{X}(x)=-\frac{T \mathcal{R}(x)}{(1+x) \mathcal{C}^{\prime}(x)} \rightarrow \frac{(1-\kappa) c \int d \tau \mathcal{M}(\tau) \tau^{1-\beta}}{(\beta-1) \int d \tau[1-\mathcal{M}(\tau)] \tau^{-\beta}}=\frac{(1-\kappa) c \int d \tau \mathcal{M}(\tau) \tau^{1-\beta}}{-\int d \tau \mathcal{M}^{\prime}(\tau) \tau^{1-\beta}}$

At first sight the value of $\mathcal{X}(x \rightarrow 0)$ appears to depend on the precise functional form of $\mathcal{M}(\tau)$. But in fact, by multiplying (301) by $\tau^{1-\beta}$ and integrating over $\tau$ one deduces

$\int d \tau \mathcal{M}^{\prime}(\tau) \tau^{1-\beta}=-c \int d \tau \mathcal{M}(\tau) \tau^{1-\beta}+\kappa \int d \tau^{\prime} \mathcal{M}\left(\tau^{\prime}\right) \int \frac{d \tau}{\tau} \frac{\tau^{1-\beta}}{1+\left(\tau^{\prime} / \tau\right)^{\beta}}$

The last $\tau$-integral evaluates to $c\left(\tau^{\prime}\right)^{1-\beta}$ and this implies from (69) that $\mathcal{X}(x \rightarrow 0)=1$ for all $\kappa$ (and $T>1 / 2$ ). Physically, this result - which can be derived similarly for Metropolis dynamics - is supported by the same intuition as for $\kappa=0$ : the initial response and decay of the correlation, and hence the FDR, are dominated by the very small fraction of histories which pass around time $t_{\mathrm{w}}$ through the shallow traps near the top of the energy landscape $(E=\mathcal{O}(1))$, where an effective pseudo-equilibrium is established.

What about long time intervals, $x \gg 1$ ? From the definition of the dynamics, it is clear that $C\left(t, t_{\mathrm{w}}\right)$ is the average of $\kappa^{j}=e^{j \ln \kappa}$ over the distribution of the number of hops $j$ between $t_{\mathrm{w}}$ and $t$. For $\kappa \approx 1$, a large number $j \sim 1 /(-\ln \kappa) \approx 1 /(1-\kappa)$ of hops is needed to get any significant decorrelation. Across the corresponding long time intervals the number of hops should average out, leading to the naive prediction $C \approx \exp \left[(\ln \kappa) \int_{t_{\mathrm{w}}}^{t} d t^{\prime} \Gamma\left(t^{\prime}\right)\right]$. Using (51), this gives the estimate

$$
\mathcal{C}(x)=(1+x)^{-\eta(1-\kappa)}, \quad \eta=c \int d \omega \omega \mathcal{P}(\omega)
$$

Intuitively, one expects that similarly $\mathcal{M}(\tau) \sim \tau^{-\eta(1-\kappa)}$ for large $\tau$. The constant $\eta$ can be evaluated explicitly, by taking the $n \rightarrow 0$ limit of (25), giving

$$
\eta=c \frac{\sin ^{2}(\pi T)}{(\pi T)^{2} \cos (\pi T)}=\frac{\tan (\pi T)}{\pi T}
$$

The analogous expression for the Metropolis case is, using (27),

$$
\eta^{\mathrm{M}}=c^{\mathrm{M}} \int d \omega \omega \mathcal{P}(\omega)=\frac{\beta}{\beta-1} \frac{(\beta-1)^{2}}{\beta(\beta-2)}=\frac{\beta-1}{\beta-2}=\frac{1-T}{1-2 T}
$$

and together with (71) confirms the estimate for the correlation function scaling in eq. (22) of [17]. Both $\eta$ and $\eta^{\mathrm{M}}$ diverge as $T \rightarrow 1 / 2$ from below, signalling a breakdown of the above naive reasoning for higher temperatures; we return to this point below. For $T \rightarrow 0, \eta$ and $\eta^{\mathrm{M}}$ both approach unity so that $\mathcal{M}(\tau)$ is predicted to decay as $\tau^{-(1-\kappa)}$, exactly as we found by exact calculation for $T=0$.

To find the asymptotics of $\mathcal{M}(\tau)$ for general $T$ and $\kappa$, one substitutes the ansatz $\mathcal{M}(\tau) \sim \tau^{-\lambda}$ into (30). The l.h.s. is then subleading and the leading terms on the r.h.s. have to cancel, giving the condition

$$
c=\kappa c_{-\lambda}
$$




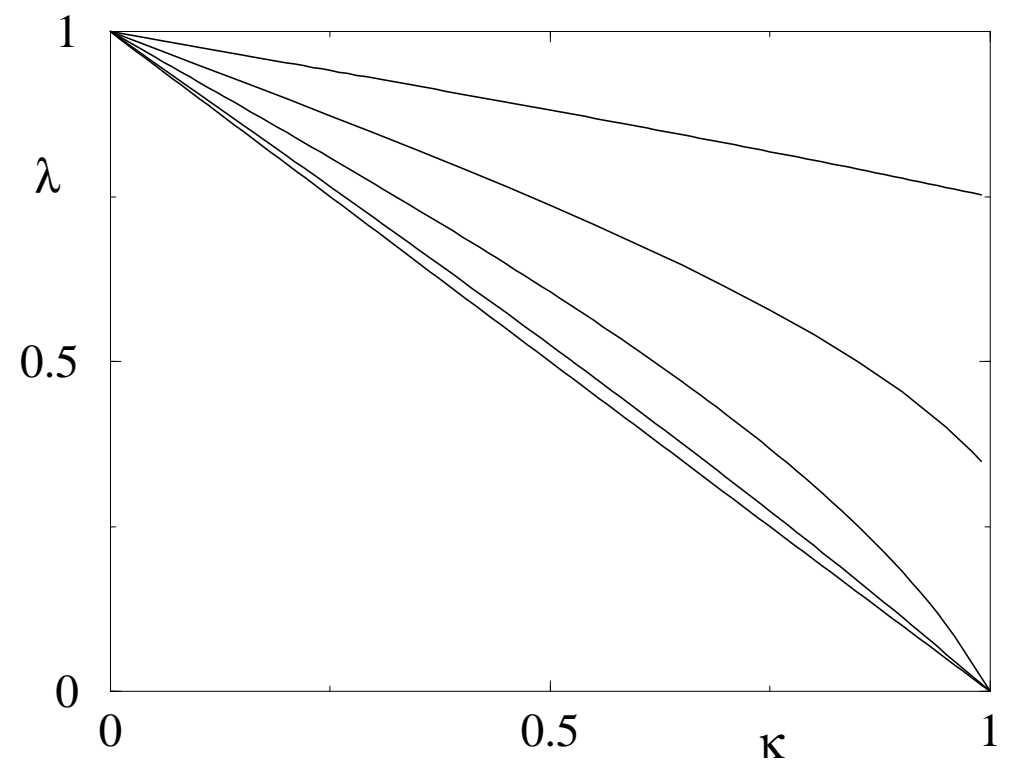

Figure 4. Calculated values of the decay exponent $\lambda$ of $\mathcal{M}(\tau) \sim \tau^{-\lambda}$ as a function of $\kappa$. The curves are for Glauber dynamics and $T=0,0.2,0.4,0.6,0.8$ from bottom to top.

for $\lambda$, or equivalently $\kappa \sin (\pi T)=\sin [\pi T(1-\lambda)]$. For $c_{-\lambda}$ to be finite one needs $\lambda<1$, and $\mathcal{M}(\tau)$ decreases with $\tau$ so $\lambda>0$. In this range of $\lambda$ the condition (174) has only one solution,

$$
\lambda=1-\frac{\arcsin [\kappa \sin (\pi T)]}{\pi T}
$$

For small $\kappa$ this exponent approaches 1 , with $\lambda=1-\kappa / c+\mathcal{O}\left(\kappa^{2}\right)$; see Fig. 4. The behaviour for $\kappa \rightarrow 1$, on the other hand, depends strongly on temperature. For $T<1 / 2$, $\lambda=(1-\kappa) \tan (\pi T) /(\pi T)=\eta(1-\kappa)$ to first order in $1-\kappa$, exactly as the naive argument had predicted. For higher temperatures $T>1 / 2$, the exponent approaches a nonzero value $\lambda=2-\beta$ in the same limit. This is rather striking since at $\kappa=1$ directly one expects $\mathcal{M}(\tau) \equiv 1$ and hence $\lambda=0$ : the limit $\kappa \rightarrow 1$ is discontinuous for $T>1 / 2$. We discuss the physical reasons for this at first sight surprising behaviour below.

The above predictions for $\lambda$ apply to the Glauber case. Using the same arguments for Metropolis rates, one is led to the condition $c^{\mathrm{M}}=\kappa c_{-\lambda}^{\mathrm{M}}$. This reads explicitly $\beta /(\beta-1)=\kappa \beta /[(1-\lambda)(\beta+\lambda-1)]$, giving

$$
\lambda=1-\frac{1}{2} \beta+\sqrt{\frac{1}{4} \beta^{2}-\kappa(\beta-1)}
$$

Similarly to the Glauber case, $\lambda \rightarrow 0$ for $\kappa \rightarrow 1$ at $T<1 / 2$, with leading order behaviour $\lambda=(1-\kappa)(\beta-1) /(\beta-2)$ in agreement with (73) . For $T>1 / 2$, on the other hand, $\lambda \rightarrow 2-\beta$ and this limit value is the same as for Glauber dynamics. In general, however, as soon as the decorrelation of the observable in a jump is not perfect $(\kappa>0)$, the decay exponents (75) for Glauber and (76) for Metropolis rates are different. This provides 
an interesting example where microscopic details of the assumed transition rates affect not only quantitative details (prefactors) but also qualitative aspects (exponents) of the dynamics.

The power-law decay of $\mathcal{M}(\tau)$ implies similar large- $x$ asymptotics for the response and correlation functions, consistent with the naive expectation explained above:

$$
\begin{aligned}
& \mathcal{C}(x) \propto x^{-\lambda} \int d \omega \omega^{-\lambda} \mathcal{P}(\omega) \\
& T \mathcal{R}(x) \propto(1-\kappa) x^{-\lambda} \int d \omega \omega^{-\lambda} r(\omega)
\end{aligned}
$$

In particular, for $T>1 / 2$ and $\kappa \rightarrow 1$, the correlation decays as $\mathcal{C}(x) \sim x^{-\lambda}$ with $\lambda=2-\beta$. This exact value is consistent with the argument by Junier and Bertin [17] that the decay exponent $\lambda$ should be close to $T=1 / \beta$ for $T \approx 1$; to first order in $1-T$ this estimate agrees with the exact prediction. It deviates progressively for lower $T$, however, with the exact value $\lambda=2-\beta$ dropping to zero at $T=1 / 2$. This is as it must be by continuity, since for $T<1 / 2$ we found that $\lambda$ vanishes for $\kappa \rightarrow 1$. As a check of our theory we have also compared the predicted exponent (76) for Metropolis dynamics with the numerical correlation function results from Fig. 9 of [17] for $\kappa=0.9$, and found very good agreement.

From the above large- $x$ behaviour of $\mathcal{C}$ and $\mathcal{R}$ we can deduce the asymptotic FDR (42) as

$$
\mathcal{X}(x \rightarrow \infty)=\frac{(1-\kappa) \int d \omega \omega^{-\lambda} r(\omega)}{\lambda \int d \omega \omega^{-\lambda} \mathcal{P}(\omega)}
$$

Using (35), one finds that the numerator integral equals $T\left[c+(1-\lambda) c_{-\lambda}\right] \int d \omega \omega^{1-\lambda} \mathcal{P}(\omega)$. The remaining moment ratio can then be calculated from (25) with $n=-\lambda$ to give

$$
\mathcal{X}(x \rightarrow \infty)=\frac{(1-\kappa) T\left[c+(1-\lambda) c_{-\lambda}\right]}{c_{-\lambda}-c}
$$

Inserting the condition (74) determining $\lambda$ simplifies this to

$$
\mathcal{X}(x \rightarrow \infty)=T(1-\lambda+\kappa)
$$

One can check that this expression is valid also for Metropolis rates, with the value of $\lambda$ then given by (76) instead of $\lambda$. For both Glauber and Metropolis rates the asymptotic FDR $\mathcal{X}(x \rightarrow \infty)$ approaches zero for $\kappa \rightarrow 0$, consistent with the results for the ordinary Barrat and Mézard model from Sec. 5. The behaviour for $\kappa \rightarrow 1$ again depends on $T$. For $T<1 / 2, \lambda \rightarrow 0$ and thus $X(x \rightarrow \infty) \rightarrow 2 T$. For $T>1 / 2$, on the other hand, $\lambda \rightarrow 2-\beta$ and $X(x \rightarrow \infty) \rightarrow 1$. Both of these values are identical to the ( $\kappa$-independent) short-time FDR $\mathcal{X}(x \rightarrow 0)$ found above. This strongly suggests that the FD plots are straight lines for $\kappa \rightarrow 1$, both above and below $T=1 / 2$. We now proceed to show this, by considering the $\operatorname{FDR} \mathcal{X}(x)$ for general $x$.

To this end, it will be useful to have an expression for $\mathcal{C}^{\prime}(x)$ that is similar in form to $\mathcal{R}(x)$. Starting from (32) one has $\mathcal{C}^{\prime}(x)=\int d \omega \mathcal{M}^{\prime}(x \omega) \omega \mathcal{P}(\omega)$. With (30) this can be written in terms of $\mathcal{M}(\cdot)$ itself as

$$
\mathcal{C}^{\prime}(x)=-c \int d \omega \mathcal{M}(x \omega) \omega \mathcal{P}(\omega)+\kappa \int d \omega \mathcal{M}(x \omega) \int d \omega^{\prime} \frac{\mathcal{P}\left(\omega^{\prime}\right)}{1+\left(\omega / \omega^{\prime}\right)^{\beta}}
$$


The $\omega^{\prime}$-integral is, from (23),$(d / d \omega)[\omega \mathcal{P}(\omega)]+c \omega \mathcal{P}(\omega)$. The second term in this sum gives a contribution of the same form as the first term on the 1.h.s. of (82), and after an integration by parts

$$
\mathcal{C}^{\prime}(x)=-c(1-\kappa) \int d \omega \mathcal{M}(x \omega) \omega \mathcal{P}(\omega)-\kappa x \int d \omega \mathcal{M}^{\prime}(x \omega) \omega \mathcal{P}(\omega)
$$

The last term is now just $-\kappa x \mathcal{C}^{\prime}(x)$ and we end up with the relatively simple expression

$$
-(1+\kappa x) \mathcal{C}^{\prime}(x)=c(1-\kappa) \int d \omega \mathcal{M}(x \omega) \omega \mathcal{P}(\omega)
$$

which also holds for Metropolis dynamics $\left(c \rightarrow c^{\mathrm{M}}\right)$. The FDR (42) then becomes a ratio of integrals involving only $\mathcal{M}(\cdot)$, rather than $\mathcal{M}(\cdot)$ and $\mathcal{M}^{\prime}(\cdot)$ as before,

$$
\mathcal{X}(x)=\frac{1+\kappa x}{1+x} \frac{\int d \omega \mathcal{M}(x \omega) r(\omega)}{c \int d \omega \mathcal{M}(x \omega) \omega \mathcal{P}(\omega)}
$$

We can now see how this simplifies for $\kappa \rightarrow 1$. For $T<1 / 2, \mathcal{M}(\tau) \rightarrow 1$ as $\kappa \rightarrow 1$ for any fixed $\tau$. The $\omega$-integrals remain finite in this limit, so that

$$
\mathcal{X}(x) \rightarrow \frac{\int d \omega r(\omega)}{c \int d \omega \omega \mathcal{P}(\omega)}=2 T
$$

exactly as in (47): $\mathcal{X}(x)=2 T$ becomes independent of $x$, and the FD plot is therefore a straight line of slope $X / T=2$. For $T>1 / 2$, on the other hand, $\mathcal{M}(\tau)$ is asymptotically a power law $\tau^{-\lambda}$, with $\lambda$ approaching $2-\beta$ from above as $\kappa \rightarrow 1$; thus for any fixed $x>0$ also $\mathcal{M}(x \omega) \sim \omega^{-\lambda}$ for large $\omega$. But $r(\omega) \sim \omega \mathcal{P}(\omega) \sim \omega^{1-\beta}$ and so the integrals in (85) become divergent at the upper end as $\kappa \rightarrow 1$. They are therefore dominated by large $\omega$-values, where $r(\omega)=c \omega \mathcal{P}(\omega)$. This shows that $\mathcal{X}(x) \rightarrow 1$ : the FD plot is again a straight line, but now of equilibrium slope $1 / T$.

With similar arguments we can also work out the correlation functions for $\kappa \rightarrow 1$. Consider $T<1 / 2$ first, and divide (84) by $\mathcal{C}(x)$ to get

$$
-(1+\kappa x) \frac{\mathcal{C}^{\prime}(x)}{\mathcal{C}(x)}=\frac{c(1-\kappa) \int d \omega \mathcal{M}(x \omega) \omega \mathcal{P}(\omega)}{\int d \omega \mathcal{M}(x \omega) \mathcal{P}(\omega)}
$$

In the limit $\kappa \rightarrow 1$ we can set $\mathcal{M} \rightarrow 1$ again and get $-d(\ln \mathcal{C}) / d(\ln (1+x))=$ $c(1-\kappa) \int d \omega \omega \mathcal{P}(\omega)=\eta(1-\kappa)$. This implies $\mathcal{C}(x)=(1+x)^{-\eta(1-\kappa)}$, exactly as the naive argument (71) suggested

For $T>1 / 2$, consider the integral on the r.h.s. of (84). This becomes divergent at the upper end for $\kappa \rightarrow 1$ as explained above, and dominated by large $\omega$. $\mathcal{M}(x \omega)$ can therefore be replaced by its asymptotic form $\sim(x \omega)^{-\lambda}$, and the r.h.s. of (84) becomes proportional to $x^{-\lambda}$, which in the limit $\kappa \rightarrow 1$ is $x^{\beta-2}$. (The remaining integral diverges as $\kappa \rightarrow 1$, but combines with the $1-\kappa$ prefactor to give a finite limit.) Thus, for $T>1 / 2$ and $\kappa \rightarrow 1$,

$$
-\mathcal{C}^{\prime}(x) \propto \frac{x^{\beta-2}}{1+x}, \quad \mathcal{C}(x)=\frac{\sin [\pi(\beta-1)]}{\pi} \int_{x}^{\infty} d x^{\prime} \frac{\left(x^{\prime}\right)^{\beta-2}}{1+x^{\prime}}
$$

In the expression for $\mathcal{C}(x)$ we have explicitly put in the proportionality constant, which follows from the equal-time value $\mathcal{C}(0)=1$. The same result also holds for Metropolis dynamics, since both the limit value of $\lambda$ for $\kappa \rightarrow 1$ and the asymptotic behaviour of $\mathcal{P}(\omega)$ and $r(\omega)$, on which the argument relies, are the same. 


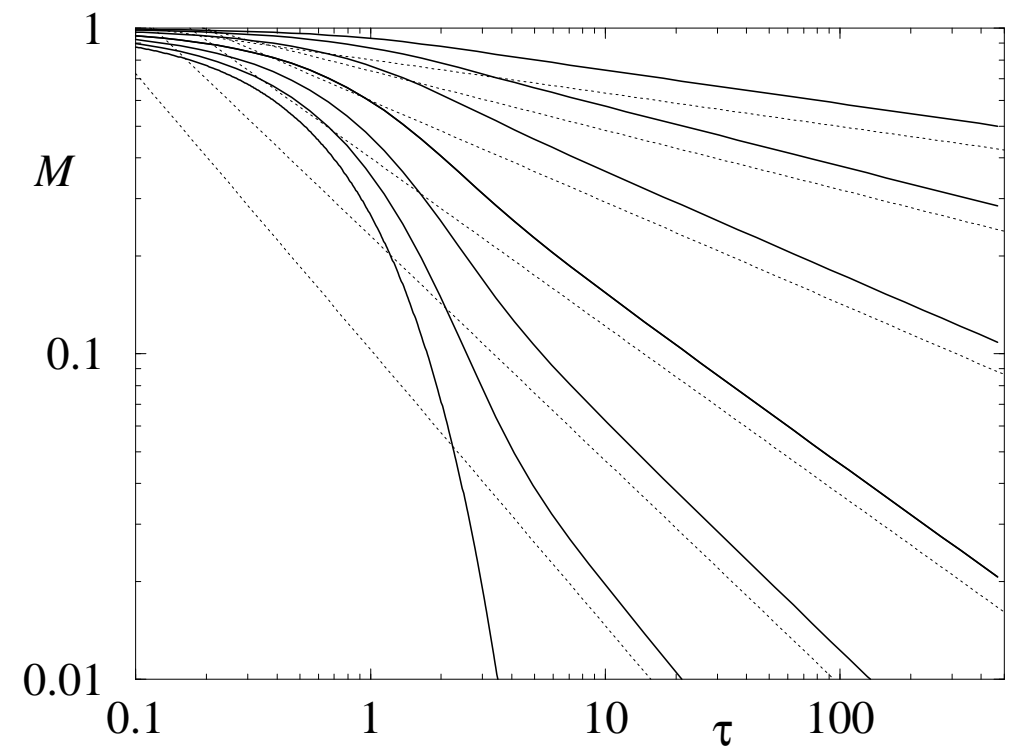

Figure 5. Calculated $\mathcal{M}(\tau)$ for $T=0.4$ and $\kappa=0$ (exact), 0.2, 0.4, 0.6, 0.8, 0.9, 0.95, from bottom to top. Dotted lines indicate the predicted $\tau^{-\lambda}$ asymptotes.

\subsection{Numerical results}

We solved (31) numerically to get $\mathcal{M}(\tau)$, using a representation which takes into account the asymptotic behaviour $\tau^{-\lambda}$, and then evaluated correlation and response. The results for $\mathcal{M}(\tau)$ at $T=0.4$ are shown in Fig. 囵, and do approach the predicted asymptotes. In particular, the slope $\lambda$ of the asymptotic power law decreases to zero for $\kappa \rightarrow 1$, while for $T=0.6$ (Fig. [6) it approaches the nonzero limit $2-\beta$. The dashed line shows the limiting form of $\mathcal{M}(\tau)$ for $\kappa \rightarrow 1$, which for $T>1 / 2$ is nontrivial. Note that we focus on Glauber dynamics throughout this section; graphs for the Metropolis case would look broadly similar as discussed above.

Fig. 17 shows the scaling function $\mathcal{C}(x)$ of the two-time correlation at $T=0.4$, again for a range of values of $\kappa$. As expected, the asymptotic decay follows the same power law as for $\mathcal{M}(\tau)$, i.e. $\mathcal{C}(x) \sim x^{-\lambda}$. In the inset we show $\mathcal{C}(x) /(1+x)^{-\lambda}$ to demonstrate that this approaches unity in the limit $\kappa \rightarrow 1$. Fig. 8 displays the analogous data for $T=0.6$. The predicted asymptotic behaviour is again observed, but now $\mathcal{C}(x)$ approaches the nontrivial limiting form for $\kappa \rightarrow 1$ predicted by (88) (dashed line).

The resulting FD plots (Fig. 9, left) for $T=0.4$ move upwards with increasing $\kappa$ as for $T=0$ and are consistent with the approach to the predicted straight line of slope 2. For $T=0.6$ (Fig. 9, right) the overall trend is similar, but the limiting straight line now has slope $1 / T$, again as predicted. 


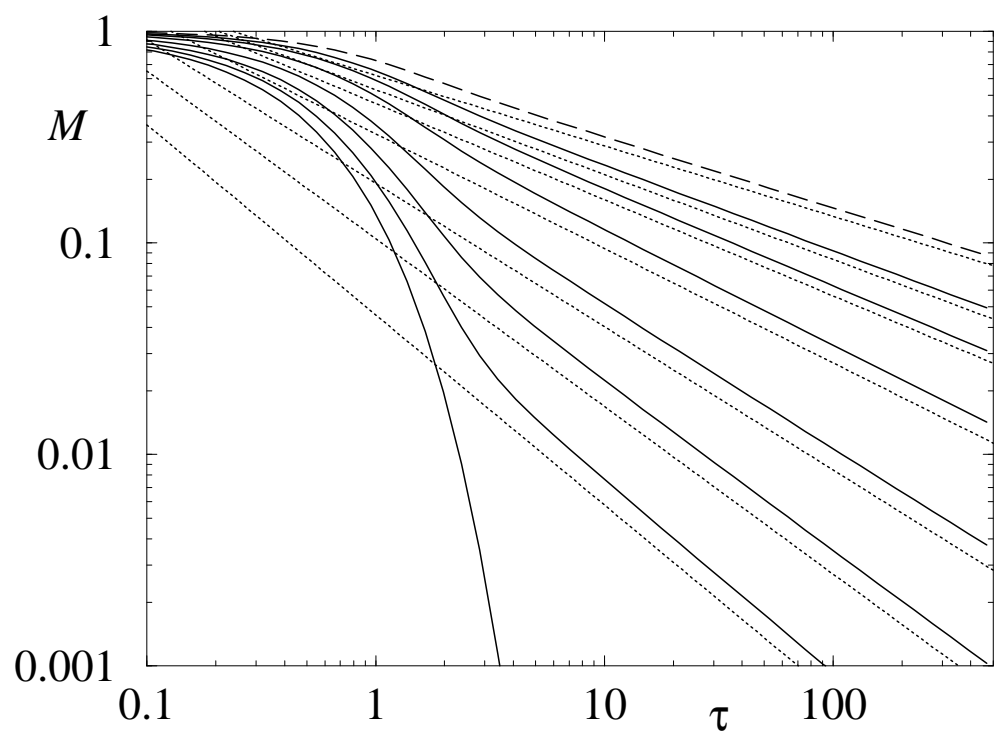

Figure 6. Analogue of Fig. 5 for $T=0.6$, for the same values of $\kappa$. The dashed line shows in addition the numerically calculated limiting form of $\mathcal{M}(\tau)$ for $\kappa \rightarrow 1$.

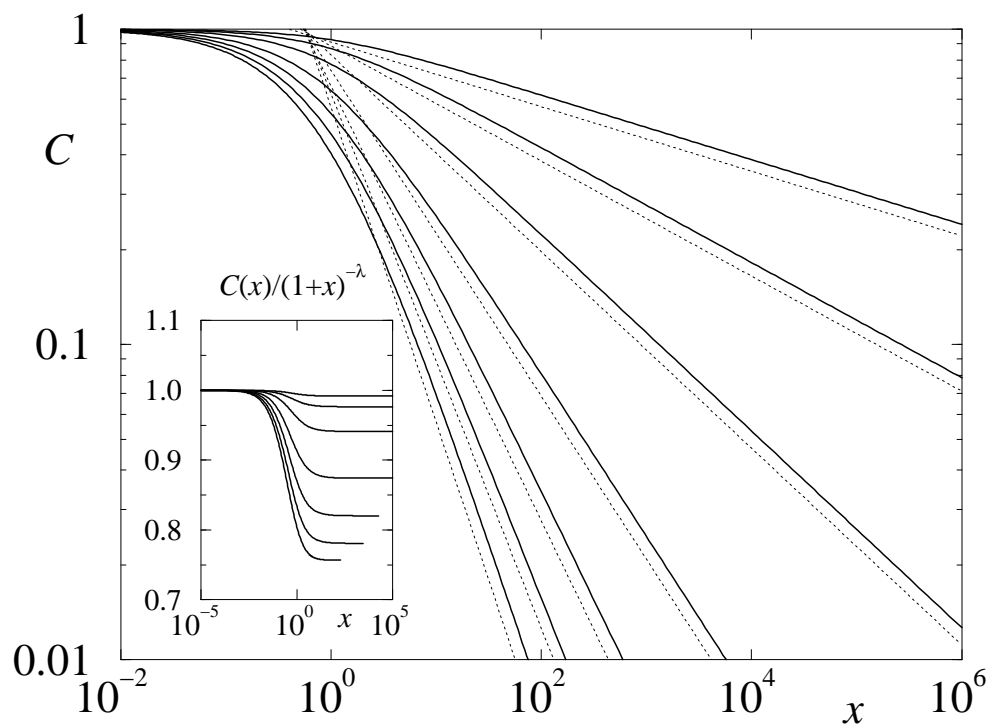

Figure 7. Correlation function $\mathcal{C}(x)$ for $T=0.4$ and the same $\kappa$ as in Fig. 5 Dotted lines indicate the predicted asymptotes $x^{-\lambda}$. The inset shows $\mathcal{C}(x) /(1+x)^{-\lambda}$ versus $x$. 


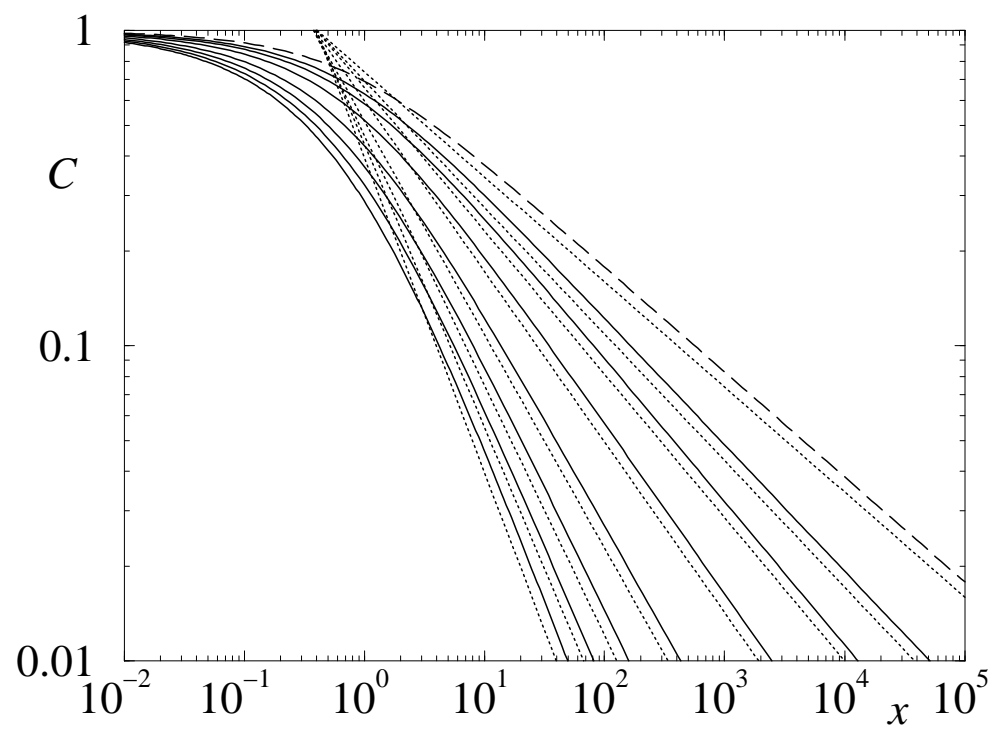

Figure 8. Correlation function $\mathcal{C}(x)$ for $T=0.6$ and the same $\kappa$ as in Fig. 6 the dashed line shows the predicted $\kappa \rightarrow 1$ limit [88). Dotted lines indicate the predicted asymptotes $x^{-\lambda}$.
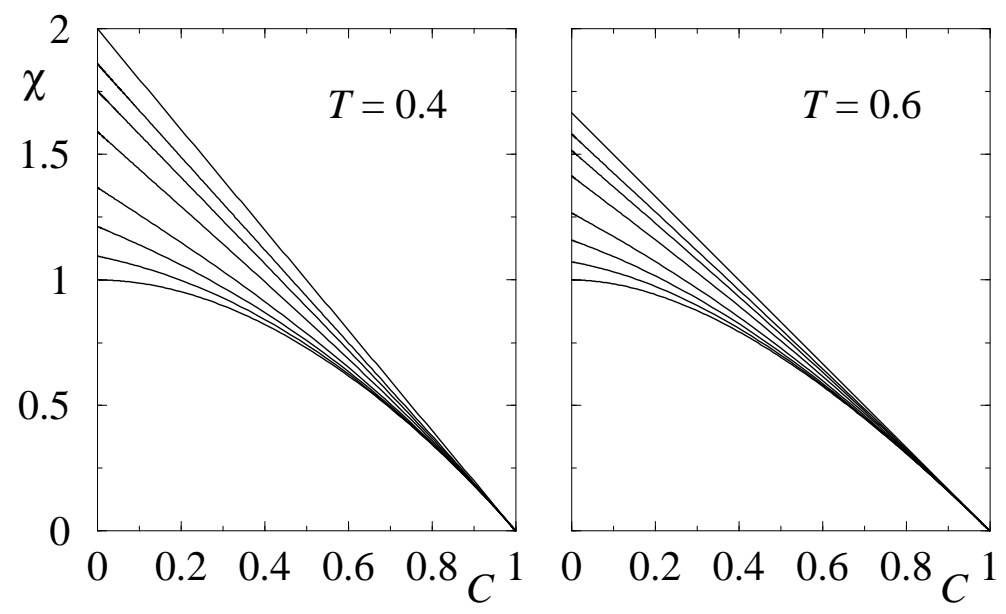

Figure 9. FD plots for $\kappa=0,0.2,0.4,0.6,0.8,0.9,0.951 .0$ (the last one being exact) from bottom to top, at temperature $T=0.4$ (left) and $T=0.6$ (right).

\subsection{Discussion}

The results found above for the limit $\kappa \rightarrow 1$ of slowly decorrelating observables provide strong support for the arguments of Junier and Bertin [17, in particular in terms of the straight-line FD plots and the asympotic decay of $\mathcal{C}(x)$ for $T<1 / 2$. In addition they clarify the limiting behaviour of $\mathcal{C}(x)$ for $T>1 / 2$, both in terms of the asymptotics $\left(\sim x^{\beta-2}\right)$ and the full (and rather simple) functional form (88). Finally, our theoretical analysis predicts how the decay exponent $\lambda$ interpolates between its value for $\kappa=0$ 
(standard Barrat and Mézard model) and the $\kappa=1$ limit of slowly decorrelating observables, and reveals that the details of this do depend on the choice of microscopic transition rates (Glauber versus Metropolis).

One of the most surprising findings is the discontinuity in the approach to $\kappa=1$ for $T>1 / 2$ : strictly at $\kappa=1$ the correlation function cannot decay and so $\mathcal{C}(x) \equiv 1$ and $\mathcal{M}(\tau) \equiv 1$. For $\kappa$ just below 1 , on the other hand, we found that these scaling functions do decay for finite values of their arguments, and approach nontrivial limit forms for $\kappa \rightarrow 1$. At first sight this seems strange: as explained above, $C\left(t, t_{\mathrm{w}}\right)$ is the average of $\kappa^{j}$ over the distribution $P_{j}\left(t, t_{\mathrm{w}}\right)$ of the number of jumps between $t_{\mathrm{w}}$ and $t$, and as $\kappa \rightarrow 1$ this average must approach unity. Indeed, this statement is true for any fixed pair of times $t$ and $t_{\mathrm{w}}$. The discontinuity only arises in the limit of large times, where $P_{j}$ consists of two essentially separate parts. The first contains finite $j$, corresponding to histories where the trap energies remain of the same order as the initial one: the system wanders among deep traps. The contribution to the correlation function arising from this part of $P_{j}$ is indeed continuous for $\kappa \rightarrow 1$. However, $P_{j}$ also has a second part (containing a finite fraction of its probability mass) at values of $j$ which are divergent with $t_{\mathrm{w}}$, presumably - for consistency with the scaling of the hopping rate - as $t_{\mathrm{w}}^{2-\beta}$. This part arises from histories which make an excursion from deep traps to the top of the landscape. In the limit $t_{\mathrm{w}} \rightarrow \infty$, the correlation decays to zero for any such history, as long as $\kappa<1$ (i.e. for any small but nonzero $1-\kappa$ ). This is why the scaling functions, which are calculated in precisely this long-time limit, are discontinuous at $\kappa=1$. For finite $t_{\mathrm{w}}$ one expects to see the crossover between correlation functions which essentially do not decay, and the limiting forms calculated for $\kappa \rightarrow 1$, to occur for $\kappa$-values with $\kappa^{t_{\mathrm{w}}^{2-\beta}}$ of order one, i.e. $1-\kappa \sim t_{\mathrm{w}}^{\beta-2}$ for large $t_{\mathrm{w}}$.

This argument also tells us how to interpret the $\kappa \rightarrow 1$ limit of $\mathcal{C}(x)$ : it is the probability of never having escaped to the shallow traps at the top of the energy landscape. It is rather intriguing to see that this can be calculated exactly while the $\kappa \rightarrow 0$ correlation, i.e. the probability of not having jumped at all, cannot as far as we are aware.

Finally, it is interesting to see what happens to our naive estimate from above, $C \approx \exp \left[(\ln \kappa) \int_{t_{\mathrm{w}}}^{t} d t^{\prime} \Gamma\left(t^{\prime}\right)\right]$, for $T>1 / 2$. This would give $C\left(t, t_{\mathrm{w}}\right)=\exp [-$ const $\times(1-$ $\left.\kappa)\left(t^{2-\beta}-t_{\mathrm{w}}^{2-\beta}\right)\right]$ which decays on subaging timescales $t-t_{\mathrm{w}} \sim t_{\mathrm{w}}^{\beta-1}$. Applied to the entire correlation function this is plainly wrong: the correlation function must decay more slowly as $\kappa$ increases, but for $\kappa=0$ we have simple aging, so $\kappa>0$ must also give simple aging or an even slower decay. We now see that the argument only applies to the decorrelation caused by large (diverging with $t_{\mathrm{w}}$ ) numbers of jumps, where it predicts correctly that the resulting contribution to the correlation function vanishes in the scaling limit. The remainder of the correlation function remains finite (and has simple aging), however, because it relates to the jumps among the deep traps which happen at rate $\sim 1 / t$, rather than to the total number of jumps which is dominated by the shallow traps. 


\section{Bouchaud model}

The dynamics of the Barrat and Mézard model can be interpreted as being slowed down by entropic barriers at low temperature: the Glauber (or Metropolis) transition rates penalize large energy increases, so that the system is forced to search for lower energy states of which there are an ever decreasing number. To complete our analysis, we now compare with the Bouchaud trap model [1, where jumps take place with rate $\exp (\beta E)$ independently of the energy of the arrival trap; thus almost all jumps return the system to the top of the energy landscape. Slow dynamics then arises purely from activation effects, i.e. the decreasing rate with which jumps from increasingly deep traps can take place. We will be interested to know in particular whether slowly decorrelating observables again produce straight-line FD plots.

Applying the intuitive reasoning from the previous section to the Bouchaud model, we see that once the system has returned to the top of the landscape it will make a large number of fast jumps, which will decorrelate the values of the observable $m$ for any $\kappa<1$. Thus, the correlation function $\mathcal{C}(x)$ should be completely independent of $\kappa$ for $0 \leq \kappa<1$ since, in the scaling limit, correlations are maintained only if no jumps at all take place $(j=0)$.

If the Bouchaud model, the natural prescription for the coupling to the field is to modify the escape rate from a trap from $\exp (\beta E)$ to $\exp [\beta(E-h m)]$ [4, 9]. This is just the original rate multiplied by $\exp (-\beta h m)$, and one is led more generally to consider an arbitrary trap model with multiplicative rates [4, 10, 11]:

$$
w\left(E^{\prime}, m^{\prime} \leftarrow E, m\right)=e^{\beta h\left[(1-\zeta) m^{\prime}-\zeta m\right]} w\left(E^{\prime} \leftarrow E\right)
$$

The notation here should be self-explanatory; the arrow points from the departure to the arrival trap. In the absence of a field all governing equations are as before, except for the replacement of $w\left(E^{\prime}-E\right)$ by $w\left(E^{\prime} \leftarrow E\right)$. The master equation then reads

$$
\frac{\partial}{\partial t} P(E, t)=-\Gamma(E) P(E, t)+\int d E^{\prime} \rho(E) w\left(E \leftarrow E^{\prime}\right) P\left(E^{\prime}, m^{\prime}, t\right)
$$

with $\Gamma(E)=\int d E^{\prime} \rho\left(E^{\prime}\right) w\left(E^{\prime} \leftarrow E\right)$, while the magnetization decay function obeys

$$
\frac{\partial}{\partial t} M(t \mid E)=-\Gamma(E) M(t \mid E)+\kappa \int d E^{\prime} M\left(t \mid E^{\prime}\right) \rho\left(E^{\prime}\right) w\left(E^{\prime} \leftarrow E\right)
$$

and the two-time correlation function is given by (9) as before. To get the response, one can start again from (15). The change in $P\left(E, m, t_{\mathrm{w}}+\Delta t\right)$ from its value without a field is given by the direct analogue of (16),

$$
\begin{aligned}
\Delta P\left(E, m, t_{\mathrm{w}}+\Delta t\right)= & \Delta t \int d E_{\mathrm{w}} d m_{\mathrm{w}} \times \\
& {\left[\rho(E) \rho\left(m \mid m_{\mathrm{w}}\right) \Delta w\left(E, m \leftarrow E_{\mathrm{w}}, m_{\mathrm{w}}\right) \rho\left(m_{\mathrm{w}}\right) P\left(E_{\mathrm{w}}, t_{\mathrm{w}}\right)\right.} \\
& \left.-\rho\left(E_{\mathrm{w}}\right) \rho\left(m_{\mathrm{w}} \mid m\right) \Delta w\left(E_{\mathrm{w}}, m_{\mathrm{w}} \leftarrow E, m\right) \rho(m) P\left(E, t_{\mathrm{w}}\right)\right]
\end{aligned}
$$

Now to linear order in $h, \Delta w\left(E, m \leftarrow E_{\mathrm{w}}, m_{\mathrm{w}}\right)=\beta h\left[(1-\zeta) m-\zeta m_{\mathrm{w}}\right] w\left(E \leftarrow E_{\mathrm{w}}\right)$. Inserting this and integrating over $m_{\mathrm{w}}$, and then over $m$ in (15), yields

$T R\left(t, t_{\mathrm{w}}\right)=\int d E d E_{\mathrm{w}} M\left(t-t_{\mathrm{w}} \mid E\right)\left\{(-\zeta \kappa+1-\zeta) \rho(E) w\left(E \leftarrow E_{\mathrm{w}}\right) P\left(E_{\mathrm{w}}, t_{\mathrm{w}}\right)\right.$ 


$$
\left.+[\zeta-(1-\zeta) \kappa] \rho\left(E_{\mathrm{w}}\right) w\left(E_{\mathrm{w}} \leftarrow E\right) P\left(E, t_{\mathrm{w}}\right)\right\}
$$

As in the case $\kappa=0$ one can now relate the response function to derivatives of the correlation function. From (9) together with (91) one has

$$
\begin{aligned}
\frac{\partial}{\partial t} C\left(t, t_{\mathrm{w}}\right)= & \int d E\left[-\Gamma(E) M\left(t-t_{\mathrm{w}} \mid E\right)\right. \\
& \left.+\kappa \int d E^{\prime} M\left(t-t_{\mathrm{w}} \mid E^{\prime}\right) \rho\left(E^{\prime}\right) w\left(E^{\prime} \leftarrow E\right)\right] P\left(E, t_{\mathrm{w}}\right)
\end{aligned}
$$

For the derivative w.r.t. the earlier time $t_{\mathrm{w}}$ one gets similarly, by also using (90),

$$
\frac{\partial}{\partial t_{\mathrm{w}}} C\left(t, t_{\mathrm{w}}\right)=(1-\kappa) \int d E d E^{\prime} M\left(t-t_{\mathrm{w}} \mid E^{\prime}\right) \rho\left(E^{\prime}\right) w\left(E^{\prime} \leftarrow E\right) P\left(E, t_{\mathrm{w}}\right)
$$

This is proportional to the first term in the expression (93) for the response, while the second term in (93) is proportional to the first one in (94), bearing in mind the definition of $\Gamma(E)$. Altogether one gets the simple relation

$$
T R\left(t, t_{\mathrm{w}}\right)=(1-\zeta)(1+\kappa) \frac{\partial}{\partial t_{\mathrm{w}}} C\left(t, t_{\mathrm{w}}\right)-[\zeta-(1-\zeta) \kappa] \frac{\partial}{\partial t} C\left(t, t_{\mathrm{w}}\right)
$$

which reduces to the known result [4, 10, 11] for $\kappa=0$ as it should and applies to all trap models with multiplicatively perturbed rates (89). At high temperatures, where a time-translation invariant equilibrium state is reached, $\partial C / \partial t_{\mathrm{w}}=-\partial C / \partial t$. As required for consistency with equilibrium FDT, the coefficients of these two quantities in (96) add up to unity. Out of equilibrium at low temperatures, an equilibrium FD relation is still recovered when the second coefficient vanishes, i.e. when $\zeta=\kappa /(1+\kappa)$. It is easy to see that this is exactly the case where the exit rate $\Gamma(E, m)$ from a given state is unperturbed by the field $h$ (to linear order), just as for $\kappa=0$ [10, 11]. This implies that for slowly decorrelating observables $(\kappa \rightarrow 1)$ one only gets a straight-line FD plot when $\zeta=1 / 2$, i.e. when the hopping rates depend on the difference $m^{\prime}-m$ as they do for Glauber dynamics.

For the Bouchaud model specifically, where $w\left(E^{\prime} \rightarrow E\right)=\exp (\beta E)$, equation (91) for $M(t \mid E)$ is easily solved by LT to give

$$
\hat{M}(s \mid E)=\frac{1}{s+e^{\beta E}}\left(1+\frac{\kappa e^{\beta E} \hat{G}(s)}{1-\kappa+\kappa s \hat{G}(s)}\right), \quad \hat{G}(s)=\int d E \frac{\rho(E)}{s+e^{\beta E}}
$$

The scaling limit for $P(E, t)$ is reached for large $t$ and low $E$ with $t e^{\beta E}$ of $\mathcal{O}(1)$. This corresponds to small $s$, of order $e^{\beta E}$. Since $\hat{G}(s) \sim s^{T-1}$ for small $s$, one sees that in this regime the second term in $\hat{M}(s \mid E)$ always becomes negligible compared to the first, as long as $\kappa<1$. This means that the $\kappa$-dependence drops out, and $M(t \mid E)=\exp \left(-t e^{\beta E}\right)$ in the scaling regime. The correlation function only picks up these scaling contributions and is therefore $\kappa$-independent as expected.

The same argument does not apply to the response, which from (96) is obviously dependent on $\kappa$. The reason is clear from (93): the contribution from $M\left(t-t_{\mathrm{w}} \mid E\right)$ is weighted with extra factors of either $\rho(E)$ or $w\left(E_{\mathrm{w}} \leftarrow E\right)\left(=e^{\beta E}\right.$ in the Bouchaud model). This means that the behaviour of $M\left(t-t_{\mathrm{w}} \mid E\right)$ for $E=\mathcal{O}(1)$ is dominant, and this does depend on $\kappa$. 


\subsection{Arguments for straight-line FD plots}

The Bouchaud trap model provides a useful point of reference from which to revisit the reasoning put forward in [17] for the emergence of straight-line FD plots in the limit $\kappa \rightarrow 1$. This has at its core the idea of considering first the change of the magnetization $m$ during $j$ jumps, and then to average over the distribution of the number of jumps between $t_{\mathrm{w}}$ and $t$. There is no problem with this for the case of the correlation function, since in the absence of a field the magnetization is just being "convected along" with the usual trap model dynamics, without itself affecting the dynamics.

For the susceptibility, on the other hand, the situation is somewhat more subtle. Consider a field switched to some nonzero value (and held there) at time $t_{\mathrm{w}}$. Then one can write generally

$$
\begin{aligned}
\chi= & \frac{\partial}{\partial h} \sum_{j} \int d m d m_{\mathrm{w}} d E_{\mathrm{w}} m P\left(m \mid j, h, E_{\mathrm{w}}, m_{\mathrm{w}}, t-t_{\mathrm{w}}\right) \times \\
& \times P_{j}\left(h, E_{\mathrm{w}}, m_{\mathrm{w}}, t-t_{\mathrm{w}}\right) P\left(E_{\mathrm{w}}, m_{\mathrm{w}}, t_{\mathrm{w}}\right)
\end{aligned}
$$

where $P\left(m \mid j, t-t_{\mathrm{w}}, h, E_{\mathrm{w}}, m_{\mathrm{w}}\right)$ is the distribution of $m$ at the end of a time interval $t-t_{\mathrm{w}}$ of constant field strength $h$, given that $j$ jumps have taken place during this time and the system started from a trap with energy $E_{\mathrm{w}}$ and magnetization $m_{\mathrm{w}} . P_{j}(\ldots)$ is the distribution of the number of jumps, which depends on the same variables. Finally, $P\left(E_{\mathrm{w}}, m_{\mathrm{w}}, t_{\mathrm{w}}\right)$ is the distribution of trap energies and magnetizations at $t_{\mathrm{w}}$, which can be written as $P\left(E_{\mathrm{w}}, t_{\mathrm{w}}\right) \rho\left(m_{\mathrm{w}}\right)$ if as we assume the field has been off up until time $t_{\mathrm{w}}$. Writing the $m$-average in (98) as $\bar{m}_{j}\left(h, E_{\mathrm{w}}, m_{\mathrm{w}}, t-t_{\mathrm{w}}\right)$ one thus has

$\chi=\frac{\partial}{\partial h} \sum_{j} \int d E_{\mathrm{w}} d m_{\mathrm{w}} \bar{m}_{j}\left(h, E_{\mathrm{w}}, m_{\mathrm{w}}, t-t_{\mathrm{w}}\right) P_{j}\left(h, E_{\mathrm{w}}, m_{\mathrm{w}}, t-t_{\mathrm{w}}\right) \rho\left(m_{\mathrm{w}}\right) P\left(E_{\mathrm{w}}, t_{\mathrm{w}}\right)$

To complete the argument of Junier and Bertin [17] two assumptions now need to be made: (A) $\bar{m}_{j}\left(h, E_{\mathrm{w}}, m_{\mathrm{w}}, t-t_{\mathrm{w}}\right)$ is the same as the average of $m$ directly after the $j$-th jump starting from a state with $E_{\mathrm{w}}$ and $m_{\mathrm{w}}$. (B) The distribution of the number of jumps $P_{j}(\ldots)$ is independent of $m_{\mathrm{w}}$, so that $\bar{m}_{j}(\ldots)$ can be averaged separately over $m_{\mathrm{w}}$. The first assumption is in general invalid because the fact that there has not been a further jump between the $j$-th jump and time $t$ favours magnetizations $m$ of the same sign as the field $h$. Indeed, in the standard $(\zeta=1, \kappa=0)$ Bouchaud model assumption (A) would imply that $\bar{m}_{j}=0$ for all $j \geq 1$ since the distribution of $m$ after any jump is unbiased. But this cannot be correct since then at long time differences, where the probability that at least one jump has taken place approaches one, the susceptibility $\chi$ would have to drop to zero. Assumption (B) is also in general not correct; e.g. in the standard Bouchaud model the jump probabilities $P_{j}$ clearly depend on the value of the starting magnetization $m_{\mathrm{w}}$ since the distribution of the time until the first jump does.

There is, however, one scenario in which these objections do not apply: if the exit rate $\Gamma(E, m)$ from a trap of energy $E$ and magnetization $m$ is independent of $h$ (and hence of $m$ ), both assumptions (A) and (B) are correct because the field biases neither the probability of not having jumped since the $j$-th jump, nor the probability of $j$ jumps 
having occurred. In the Bouchaud model, this field-independence of the exit rate holds when $\zeta=\kappa /(1+\kappa)$. One then works out easily, by considering how the Gaussian distribution of $m$ changes with every jump, that

$$
\bar{m}_{j}(\ldots)=\kappa^{j} m_{\mathrm{w}}+\beta h(1-\zeta)(1+\kappa)\left(1-\kappa^{j}\right)
$$

The susceptibility is then $\chi=\beta(1-\zeta)(1+\kappa)\left\langle 1-\kappa^{j}\right\rangle=\beta\left\langle 1-\kappa^{j}\right\rangle$, where the average is over the distribution of jumps $\int d E_{\mathrm{w}} P_{j}\left(E_{\mathrm{w}}, t-t_{\mathrm{w}}\right) P\left(E_{\mathrm{w}}, t_{\mathrm{w}}\right)$. Since the correlation function is $C=\left\langle\kappa^{j}\right\rangle$, it follows that $T \chi=1-C$ and one has equilibrium FD behaviour. This is entirely consistent with the exact relation (96).

In the Barrat and Mézard model, assumption (A) can still be justified for $\kappa \rightarrow 1$ : one can show [17] that the exit rates are affected by the field via terms of $\mathcal{O}((1-\kappa) h)$, which vanish in the limit of slow decorrelation. One is then allowed to find $\bar{m}_{j}$ from the distribution of $m$ directly after the $j$-th jump, giving at low temperatures $\bar{m}_{j}(\ldots)=\kappa^{j} m_{\mathrm{w}}+2 h\left(1-\kappa^{j}\right)$ [17. However, for $\kappa \rightarrow 1$ one needs a number of jumps $j=\mathcal{O}(1 /(1-\kappa))$ to see any significant response of the system, and similarly any significant decay of the correlation function. It is then not clear that assumption (B) can still be justified, since the small $O(1-\kappa)$ changes of each exit rate accumulated over this many jumps could still add up to a nontrivial $m_{\mathrm{w}}$-dependence of $P_{j}\left(t, h, E_{\mathrm{w}}, m_{\mathrm{w}}, t_{\mathrm{w}}\right)$.

We note finally that, for $T>1 / 2$ and $\kappa \rightarrow 1$ in the Barrat and Mézard model, one can state the argument for a straight-line FD plot without actually requiring assumption (B): histories with a finite number of jumps $j$ do not contribute to $\chi$ because for $\kappa \rightarrow 1$ the magnetization remains pinned to its initial value, whatever the field $h$. On the other hand, histories with diverging $j$ always give the equilibrium susceptilibity because they pass through the equilibrated shallow traps at the top of the landscape. Because $C$ is just the probability of $j$ being finite as argued in Sec. 7.2, this gives the equilibrium FD relation $\chi=\chi_{\mathrm{eq}}(1-C)=\beta(1-C)$.

\section{Conclusion}

We have studied trap models with observables that decorrelate slowly, by a factor $\kappa \approx 1$ with each jump. Our motivation was to clarify and extend with an analytical study the interesting observations of Junier and Bertin for a spin model which leads to trap model dynamics of this type 17. Our analysis showed that in the limit of long times, correlation and response functions are determined by two scaling functions: $\mathcal{P}(\omega)$, which gives the scaling of the distribution across trap energies, and $\mathcal{M}(\tau)$, which depends on $\kappa$ and encapsulates the decay of the observable with scaled time. Both of these are determined by integro-differential equation which can be solved exactly at $T=0$ and by appropriate numerical iteration procedures otherwise.

We focussed mostly on the Barrat and Mézard trap model which has entropic barriers that slow its dynamics at low temperature. In Sec. 5 we considered this first for fast decorrelation, $\kappa=0$, to extend previous results which had been limited to $T=0$. The results already reveal changes in the dynamics at $T=1 / 2$, i.e. half the 
glass transition temperature $T_{\mathrm{g}}=1$ of the model: for $T<1 / 2$ the aging behaviour is simple, with the average hopping rate scaling as the inverse of the age, while for $T>1 / 2$ a power law scaling obtains which is dominated by rare excursions of the system to the top of its energy landscape, i.e. to the shallow traps. Correspondingly the initial slope of the FD plot of susceptibility versus correlation is $1 / T_{\text {eff }}$ with $T_{\text {eff }}=1 / 2$ for $T<1 / 2$, and $1 / T$ for larger temperatures. The long-time susceptibility $\chi\left(t, t_{\mathrm{w}}\right)$ for $t \gg t_{\mathrm{w}}$, i.e. for $C\left(t, t_{\mathrm{w}}\right) \rightarrow 0$, remains constant throughout the glassy region $T<1$, effectively "freezing" to its value at $T=1$ as temperature is lowered through the glass transition.

In Sec. [ 6 we looked at the complementary case of $T=0$ but slow decorrelation, $\kappa>0$. Here all scaling functions can be found exactly. The initial slope of the FD plot, $1 / T_{\text {eff }}=2$, remains $\kappa$-independent, while the asymptotic (for $C \rightarrow 0$ ) slope $2 \kappa$ grows linearly with $\kappa$. In the limit $\kappa \rightarrow 1$ of slow decorrelation the two slopes coincide, and the FD plot becomes a straight line.

Finally, in Sec. 7 we extended the analysis to general decorrelation $(\kappa>0)$ and nonzero temperature. Scaling functions now need to be found numerically, but we were able to predict the exponents for their power-law decays in closed form. Surprisingly, these exponents depend on whether Glauber or Metropolis transition rates are used, contrary to the usual expectation that such choices only have minor quantitative effects. We were able to show explicitly that the initial slopes of the FD plots are $\kappa$-independent, and that in the limit $\kappa \rightarrow 1$ of slow decorrelation the FD plots become straight lines as argued qualitatively by Junier and Bertin [17]. Their slopes, coinciding as they must with the $\kappa$-independent initial slopes, correspond to FD relations with an effective temperature $T_{\text {eff }}=1 / 2$ for $T<1 / 2$ and equilibrium FDT for $T>1 / 2$. We discussed in detail the approach to the limit $\kappa \rightarrow 1$; for $T>1 / 2$ this is discontinuous because rare excursions to the top of the landscape achieve full decorrelation (and an associated equilibrium response) whenever $\kappa$ is below unity by a nonzero amount $1-\kappa$.

We generalized to the Bouchaud trap model, and more broadly any trap model with rates that are multiplicatively perturbed by the applied field, in Sec. 8 . Here it turns out that straight-line FD plots always have an equilibrium slope of $1 / T$. However, they do not necessarily arise even in the limit $\kappa \rightarrow 1$, but rely on a specific assignment of the field-dependence of the transition rates which eliminates the effect of the field on the residence time in a given trap. This example illustrated that simple arguments for the existence of straight-line FD relations require some caution because the implicit assumptions can be difficult to justify.

Looking at our results more broadly, it is intriguing to see that slowly decorrelating observables can produce non-trivial straight-line FD plots in trap model, something which can otherwise be achieved only by choosing rates that violate detailed balance [10]. The significance of this is that such plots indicate that the effective temperature is (in the long-time, non-equilibrium regime) independent of the pair of observation times; this is one of the plausible requirements for $T_{\text {eff }}$ to be a physically meaningful quantity. What remains unclear to us is why the notional "glass transition" temperature which slowly decorrelating observables seem to measure in trap models is half the usual 
glass transition temperature $T_{\mathrm{g}}$ ( $=1$ in our units). One way of interpreting this result [17] is that slowly decorrelating observables only pick up non-equilibrium effects caused by entropic rather than energetic barriers. Alternatively, one could argue that slowly decorrelating observables detect only persistently slow dynamics. As soon as the system begins to exhibit intermittent behaviour, where episodes of fast dynamics in the effectively equilibrated parts of phase space alternate with periods of slow wandering around regions of low energy, effective equilibrium FD relations are recovered. Slowly decorrelating observables can thus help us to single out parts of the dynamics which can be meaningfully associated with an effective temperature, but by the same token can be blind to other aspects of the dynamics that remain clearly out of equilibrium. How this trade-off operates in other glassy systems is certainly worthy of further study. One challenge will be to integrate finite spatial dimensionality into the picture. One expects, for example, that the dynamics will then effectively decompose into that of independent subsystems whose finite size is set by a time-dependent correlation length [25]. Decorrelation can then not be arbitrarily slow: if each subsystem contains $N_{\text {eff }}$ degrees of freedom, say, one would expect $\kappa<1-\mathcal{O}\left(1 / N_{\text {eff }}\right)$. The limit of slow decorrelation we focussed on here would then require relatively large correlated subsystems.

Acknowledgements: We are grateful to Eric Bertin, Ivan Junier and Felix Ritort for helpful and inspiring discussions.

\section{References}

[1] J P Bouchaud. Weak ergodicity breaking and aging in disordered-systems. J. Phys. (France) I, 2(9):1705-1713, 1992.

[2] A Barrat and M Mézard. Phase-space diffusion and low-temperature aging. J. Phys. (France) I, 5(8):941-947, 1995.

[3] J. C. Dyre. Master-equation approach to the glass transition. Phys. Rev. Lett., 58:792, 1987.

[4] J P Bouchaud and D S Dean. Aging on Parisi's tree. J. Phys. (France) I, 5(3):265-286, 1995.

[5] J P Bouchaud, A Comtet, and C Monthus. On a dynamical model of glasses. J. Phys. (France) $I, 5(12): 1521-1526,1995$.

[6] C Monthus and J P Bouchaud. Models of traps and glass phenomenology. J. Phys. A, 29(14):38473869, 1996.

[7] D Head. Zero-temperature criticality in a simple glass model. J. Phys. A, 33(3):465-478, 2000.

[8] E Bertin and J P Bouchaud. Dynamical ultrametricity in the critical trap model. J. Phys. A, 35(13):3039-3051, 2002.

[9] S Fielding and P Sollich. Observable dependence of fluctuation-dissipation relations and effective temperatures. Phys. Rev. Lett., 88:050603, 2002.

[10] F Ritort. Universal dependence of the fluctuation-dissipation ratio on the transition rates in trap models. J. Phys. A, 36(43):10791-10805, 2003.

[11] P Sollich. Fluctuation-dissipation relations in trap models. J. Phys. A, 36(43):10807-10818, 2003.

[12] J W Haus and K W Kehr. Diffusion in regular and disordered lattices. Phys. Rep., 150(5-6):263406, 1987.

[13] B Rinn, P Maass, and J P Bouchaud. Multiple scaling regimes in simple aging models. Phys. Rev. Lett., 84(23):5403-5406, 2000. 
[14] B Rinn, P Maass, and J P Bouchaud. Hopping in the glass configuration space: subaging and generalized scaling laws. Phys. Rev. B, 64:104417, 2001.

[15] E M Bertin and J P Bouchaud. Linear and nonlinear response in the aging regime of the onedimensional trap model. Phys. Rev. E, 67:065105, 2003.

[16] I Junier and J Kurchan. Microscopic realizations of the trap model. J. Phys. A, 37(13):3945-3965, 2004.

[17] I Junier and E Bertin. Dynamic phase diagram of the number partitioning problem. Phys. Rev. E, 70:066126, 2004.

[18] L F Cugliandolo and J Kurchan. Analytical solution of the off-equilibrium dynamics of a longrange spin-glass model. Phys. Rev. Lett., 71(1):173-176, 1993.

[19] L F Cugliandolo and J Kurchan. On the out-of-equilibrium relaxation of the SherringtonKirkpatrick model. J. Phys. A, 27(17):5749-5772, 1994.

[20] L F Cugliandolo, J Kurchan, and L Peliti. Energy flow, partial equilibration, and effective temperatures in systems with slow dynamics. Phys. Rev. E, 55(4):3898-3914, 1997.

[21] A Crisanti and F Ritort. A glass transition scenario based on heterogeneities and entropy barriers. Philos. Mag. B, 82(2):143-149, 2002.

[22] E M Bertin. Cross-over from entropic to thermal dynamics in glassy models. J. Phys. A, 36(43):10683-10700, 2003.

[23] P Sollich, S Fielding, and P Mayer. Fluctuation-dissipation relations and effective temperatures in simple non-mean field systems. J. Phys. Cond. Matt., 14(7):1683-1696, 2002.

[24] P Mayer, L Berthier, J P Garrahan, and P Sollich. Fluctuation-dissipation relations in the nonequilibrium critical dynamics of Ising models. Phys. Rev. E, 68:016116, 2003.

[25] A Heuer, B Doliwa, and A Saksaengwijit. Potential-energy landscape of a supercooled liquid and its resemblance to a collection of traps. Phys. Rev. E, 72:021503, 2005. 